\title{
氟硼二吡咯亚甲基类荧光探针在离子检测中的应用进展
}

\author{
何源冯若昆易云瑞刘占祥* \\ (浙江大学化学系 杭州 310007)
}

\begin{abstract}
摘要 氟嗍二吡咯亚甲基类(BODIPY)类化合物具有摩尔吸光系数高、荧光量子产率高、吸收波长在可见光或近红外区 域、苂光寿命长和光稳定性好等特点，并且其荧光对溶剂的极性和 $\mathrm{pH}$ 均不敏感，是一类可应用于生物领域的荧光染料， 近年来，此类化合物被广泛用于设计合成荧光传感器分子，用于各种离子的检测，此类苂光探针具有分析灵敏度高、选 择性好等特点. 综述了氟嗍二吡咯亚甲基类荧光探针在离子识别和检测中的应用，并展望了该领域的前景.

关键词＼cjkstart氟硼二吡咯亚甲基(BODIPY)；荧光传感器；选择性识别；离子探针
\end{abstract}

\section{Recent Progress in the Research of Borondipyrromethene-Based Fluorescent Ion Chemosensor}

\author{
He, Yuan Feng, Ruokun Yi, Yunrui Liu, Zhanxiang* \\ (Department of Chemistry, Zhejiang University, Hangzhou 310007)
}

\begin{abstract}
Borondipyrromethene (BODIPY) derivatives have been widely exploited and recognized as useful fluorescent sensing and labeling reagents in biomolecules due to their unique photophysical properties, such as strong absorption in the visible and near-IR ranges, high fluorescence quantum yield and excellent photo-stability. Recently, many BODIPY-derived probes have been developed for the detection of metal ions. BODIPY dye as a kind of fluorescent probe has been obtained much attention, which has outstanding physicochemical properties and excerlent selectivity, and sentiivity. In this review, the progress in the fields of ion chemosensor based on BODIPY is summarized.
\end{abstract}

Keywords borondipyrromethene (BODIPY); fluorescent sensor; selective recognition; ion chemosensor

近年来，苂光检测技术的快速发展使细胞乃至活体 苂光成像成为可能. 荧光检测技术具有灵敏度高、选择 性好、成本低、易操作以及适用面广等特点, 目前已在 生物分析领域占有一席之地. 苂光检测技术的核心在于 高性能的苂光染料, 其性能的优劣直接影响检测的准确 度和灵敏度, 因此寻找性能优异的苂光染料对于生物分 析技术的发展具有重要意义.

氟嗍二吡咯亚甲基 (Borondipyrromethene，简称 BODIPY) 是 20 世纪 60 年代发展起来的一类小分子荧光 染料. 1968 年 Treibs 和 Kreuzer ${ }^{[1]}$ 首次合成了氟嗍二吡咯 亚甲基类荧光染料，其母体结构如图 1 所示.

由于 BODIPY 具有其它荧光染料所不具备的优良 光学性质, 如(1)很高的摩尔消光系数; (2)较高的苂光量 子产率, 能量转换效率高; (3)很强的光和化学稳定性,

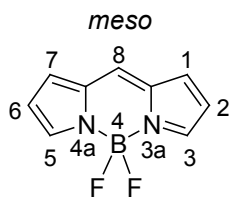

图 1 BODIPY 母体结构

Figure 1 BODIPY core structure

对溶剂极性和环境 $\mathrm{pH}$ 几乎不敏感; (4)荧光光谱峰宽较 窄，分析灵敏度高; (5)染料分子中不含电荷，可避免与 其它离子之间的静电作用，因此近年来被广泛应用于生 物苂光分析领域 ${ }^{[2 a, 2 b]}$. 近年来也有对此类化合物不同应 用的详细报道 ${ }^{[2 c ~ 2 e]}$. 本文综述了 2011 年以来 BODIPY 类苂光探针在离子检测领域的应用的新方法与新进展, 并展望了其发展前景.

\footnotetext{
*E-mail: liuzhanx@zju.edu.cn

Received March 30, 2014; revised June 13, 2014; published online July 1, 2014.

Project supported by the National Natural Science Foundation of China (No. 20802068) and the Natural Science Foundation of Zhejiang Province (No. Y4110100).

国家自然科学基金(No. 20802068)、浙江省自然科学基金(No. Y4110100)资助项目.
} 


\section{BODIPY 类荧光探针的设计原理}

在对 BODIPY 基本结构单元进行化学修饰后，可以 使其成为能在生物体内实现特定功能的荧光探针. 荧光 探针主要由识别基团、连接臂和荧光发色团等结构单元 组成. 在识别检测对象时, 荧光发色基团的光物理性质 受到影响, 苂光信号的输出形式发生改变, 例如苂光峰 值位置的移动, 荧光量子产率的涨落以及新荧光峰的出 现等, 因此苂光团可起到信息转化的作用, 即将识别信 息转换为荧光信号, 这一转化过程中涉及的机理主要有 光致电子转移(Photoinduced Electron Transfer, PET)、分 子内电荷转移(Intramolecular Charge Transfer, ICT)和荧 光共振能量转移 (Fluorescence Resonance Energy Transfer, FRET)等，基本原理如图(图 2４)所示:

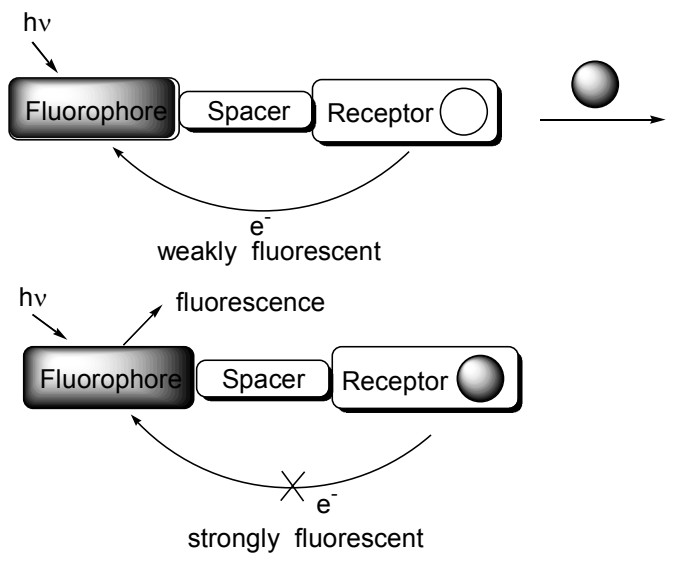

图 2 PET 探针荧光机理

Figure 2 “Turn-on” sensing mechanism of PET sensors
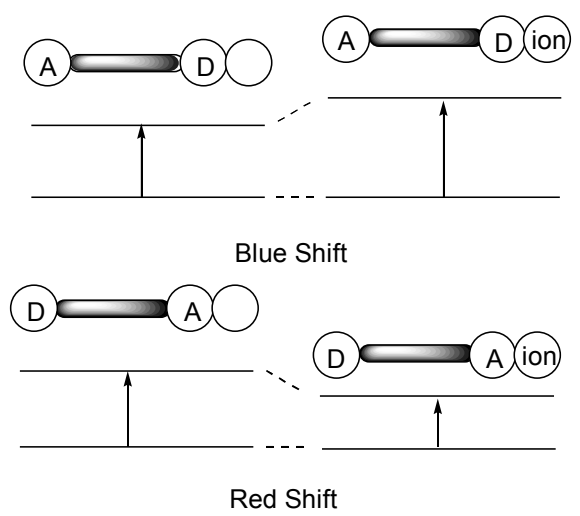

图 3 ICT 荧光传感器一般原理示意图

Figure 3 ICT fluorescent sensors and sensing mechanisms

\section{BODIPY 类荧光探针对阳离子的检测}

\subsection{BODIPY 类荧光探针检测 $\mathrm{H}^{+}$}

由于细胞内的生化反应进程十分复杂, 测定活细胞 内的酸碱度变化具有极大的挑战性. 2009 年 Burgess

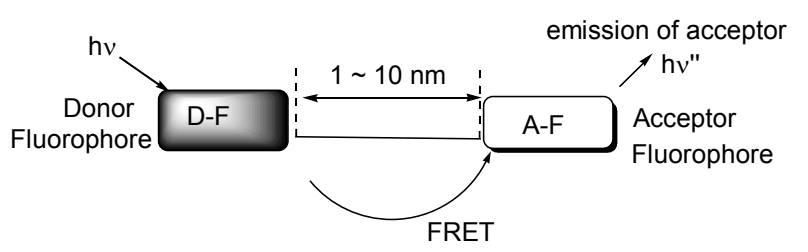

图4 FRET探针苂光机理

Figure 4 Fluorescence mechanism of FRET sensors

等 ${ }^{[3]}$ 实现了在 $488 \mathrm{~nm}$ 可见光激发下苂光探针对 COS-7 细胞内 $\mathrm{pH}$ 值的检测. 该探针以 BODIPY 为核心, 在 2,6 号位上连接 2 个苂光素基团，当 $\mathrm{pH}$ 值变化时，苂光素上 的氧杂葋会以酚盐或酚的形式存在. 当 $\mathrm{pH}<6.0$ 时，以 酚形式存在的荧光素基团与 BODIPY 之间发生共振能 量转移, BODIPY 发射红色荧光, 波长约 $600 \mathrm{~nm}$ (图 5); 而 $\mathrm{pH}>7.0$ 时, 菼光素以酚氧离子的形式存在, 共振能 量转移受阻, 荧光素发射绿色荧光, 波长约 $525 \mathrm{~nm}$ (图 $3) ; \mathrm{pH}$ 值在 6 7 之间时, 则通过测定红色荧光和绿色荧 光强度之比检测 $\mathrm{pH}$, 成为目前首例将荧光共振能量转 移机理应用于细胞内 $\mathrm{pH}$ 检测的比率苂光探针. 由于水 溶性良好，该探针还可通过共价键连接到特定蛋白质分 子上, 通过荧光对蛋白质分子进行追踪, 实现对细胞内 的生化反应过程的监控.

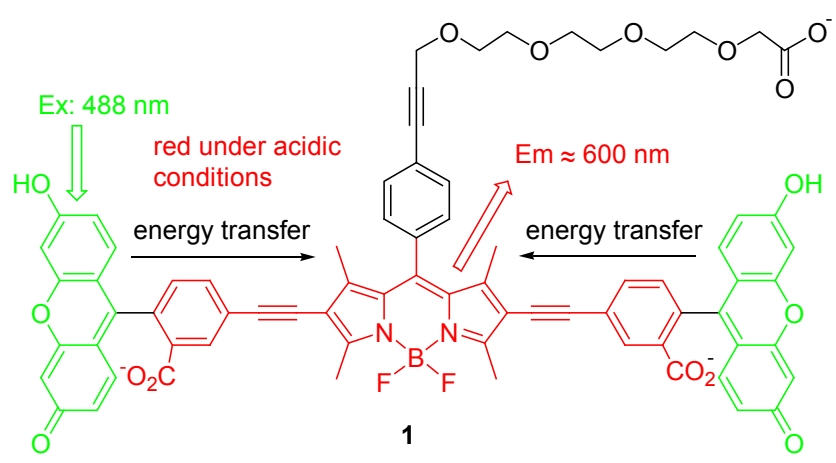

图 5 探针 1 在酸性条件下的苂光机理

Figure 5 Sensing mechanism under acidic condition

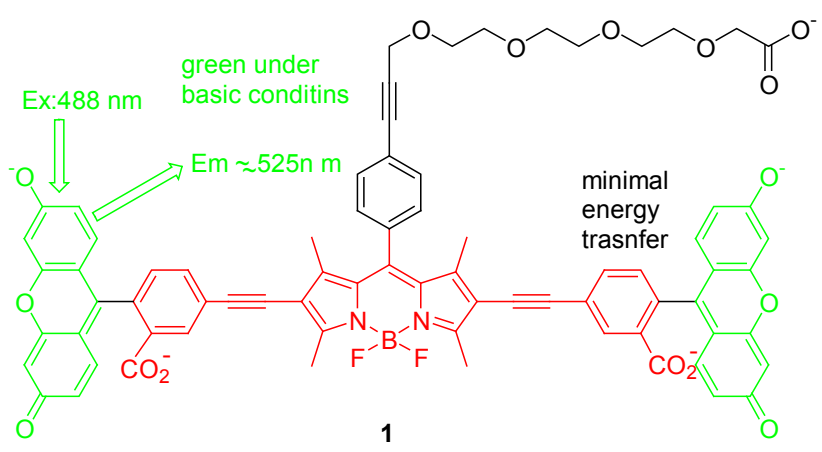

图 6 探针 1 碱性条件下的苂光机理

Figure 6 Sensing mechanism under basic condition 
2011 年 Boens 等 ${ }^{[4]}$ 利用咪唑基团为 $\mathrm{H}^{+}$识别体，报道 了两种新型近中性 $\mathrm{pH}$ 探针(图 7). 探针 2 具有良好的水 溶性, 当 $\mathrm{pH}$ 从 4.5 升至 7.0 时, 苂光发射波长由 $558 \mathrm{~nm}$ 红移至 $570 \mathrm{~nm}$, 颜色由黄绿转为橘黄, 碱性条件下的光 谱性质是在纯水中测试的, 当 $\mathrm{pH}$ 从 10.75 升至 12.54 时, $570 \mathrm{~nm}$ 处的荧光峰强度逐渐下降. 此外, 探针 3 能够很 好地穿透细胞膜, 并在细胞质中水解为探针 $\mathbf{2}$, 实现了 HEK293 细胞中的苂光共聚焦成像.

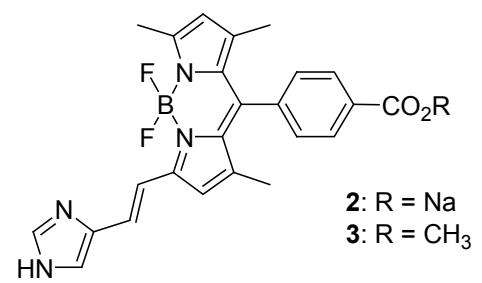

图 7 BODIPY 近中性 $\mathrm{pH}$ 荧光探针 $\mathbf{2}$ 和 3

Figure 7 BODIPY near-neutral $\mathrm{pH}$ sensor

同年 Chen 等 ${ }^{[5]}$ 利用 8 -着基喹啉既是弱酸又是弱碱 的性质，在 BODIPY 骨架上引入 8-美基喹啉作为检测基 团, 得到基于 PET 机理的 OFF-ON-OFF 型 $\mathrm{pH}$ 探针 $\mathbf{4}$ (Scheme 1). 在酸性环境中, 喹啉上的 $\mathrm{N}$ 原子质子化, 产 生从 BODIPY 到喹啉的 PET 作用, 荧光被淬灭; 在中性 环境中探针的苂光恢复正常; 而在碱性环境中喹啉上的 差基去质子化, 形成由啥啉到 BODIPY 的 PET 作用, 苂 光被淬灭. 因此在 $\mathrm{pH}$ 值递增时, 该探针呈现苂光强度 的 OFF-ON-OFF 转变. 与先前报道的其它探针相比, 该 探针的检测范围大大增加, $\mathrm{pH}$ 响应范围达到 $0.49 \sim 13.1$.

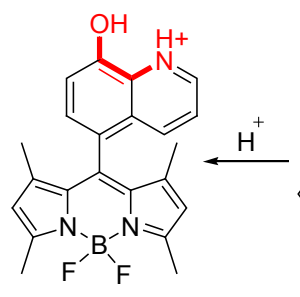

OFF

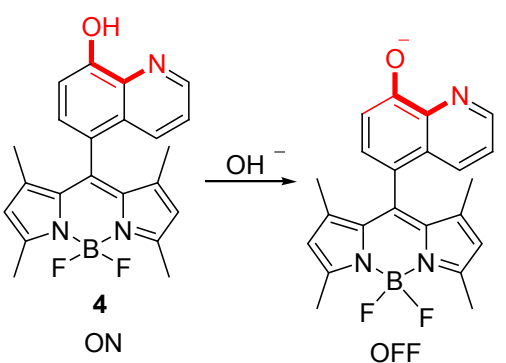

Scheme 1
$\mathrm{pH}$ 的精确测定对于生物学研究具有十分重要的意 义, 而传统的玻璃电极无法对极端 pH 进行检测. 2013

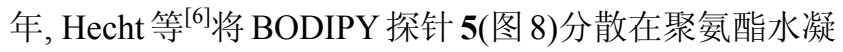
胶中, 再附着在环氧树脂基底上而形成 “ $\mathrm{pH}$ 检测计” . 该检测计的动态响应 $\mathrm{pH}$ 范围为 $10.0 \sim 13.1$, 且能够在 高离子活度的溶液中工作. 碱性阴离子对探针几乎没有 影响，荧光信号对 $\mathrm{pH}$ 的变化完全可逆.

\subsection{BODIPY 荧光探针检测 $\mathrm{Zn}^{2+}$}

$\mathrm{Zn}^{2+}$ 作为生命体系中众多的微量元素之一, 是细胞

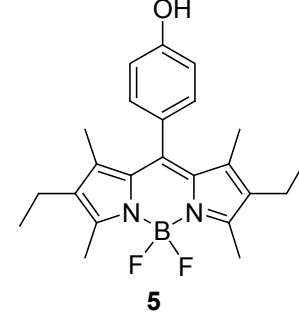

图 8 强碱性 BODIPY pH 探针 5

Figure 8 BODIPY pH sensor 5 working in highly basic solution

内很多酶和转录因子的重要组成部分，可能是细胞新陈 代谢的主要调控因子, 在神经递质传递过程中起着重要 作用，因此，发展特异性识别 $\mathrm{Zn}^{2+}$ 的传感器尤为重要. 基于席夫碱能很好地螯合过渡金属离子并延伸共轭体 系, 2012 年 $\mathrm{CaO}$ 等 ${ }^{[7]}$ 设计合成了发射波长位于近红外区 的 $\mathrm{Zn}^{2+}$ 开关型探针 6 (图 9). $\mathrm{Zn}^{2+}$ 与该探针在 $\mathrm{CH}_{3} \mathrm{CN}$ 中 以 $1: 2$ 螯合后, $680 \mathrm{~nm}$ 处苂光量子产率由 0.006 升至 0.19 , 苂光增强约 32 倍，溶液颜色由浅紫变为浅蓝，实 现了对 $\mathrm{Zn}^{2+}$ 的比色-荧光双重检测. 有趣的是，该探针 在 $V\left(\mathrm{CH}_{3} \mathrm{CN}\right): V($ Tris-HCl $)=1: 1$ 溶液中表现出对 $\mathrm{Zn}^{2+}$ 极佳的选择性，即使是 $\mathrm{Cd}^{2+}$ 也无法对检测形成干扰.

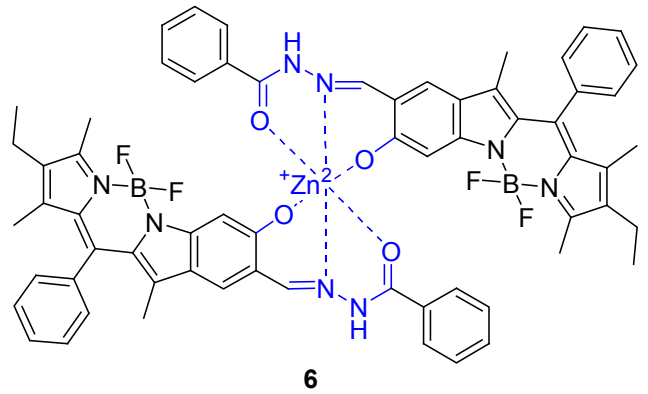

图 9 近红外 BODIPY $\mathrm{Zn}^{2+}$ 探针 6

Figure 9 NIR BODIPY $\mathrm{Zn}^{2+}$ sensor 6

同年, Kang 等 ${ }^{[8]}$ 将 BODIPY 探针负载到 $\mathrm{Fe}_{3} \mathrm{O}_{4}$ 纳米 粒子表面, 得到了一种基于 PET 机理的水溶性探针-纳 米粒子复合体 7 (Eq. 1). 在 HEPES 缓冲溶液中, $\mathrm{Zn}^{2+}$ 的 加入阻断了 PET 作用, $594 \mathrm{~nm}$ 处的苂光增强了约 32 倍, 常见金属阳离子对探针没有干扰. BPDIPY 探针本身并 不溶于水, 在 $\mathrm{CH}_{3} \mathrm{CN}$ 中加入 $\mathrm{Zn}^{2+}$ 后苂光仅增强 1.5 倍, $\mathrm{Al}^{3+}$ 等均能干扰识别过程. 探针复合体荧光强度在 0 $30 \mu \mathrm{g} / \mathrm{L}$ 范围内与 $\mathrm{Zn}^{2+}$ 浓度成正比, 检测下限达到了 0.2 $\mathrm{nmol} / \mathrm{L}$, 响应时间小于 $1 \mathrm{~min}$. 在 $\mathrm{pH}=3 \sim 11$ 范围内, 空 白探针始终没有苂光，但探针复合体的荧光却受到 $\mathrm{pH}$ 影响, $\mathrm{pH}=7.4$ 时荧光强度达到最大. 探针在 $\mathrm{HeLa}$ 细胞 中实现了对 $\mathrm{Zn}^{2+}$ 的苂光标记.

设计具有良好水溶性且发射波长位于近红外区的 探针对于生物检测具有极其重要的意义. 2013 年, Zhu 


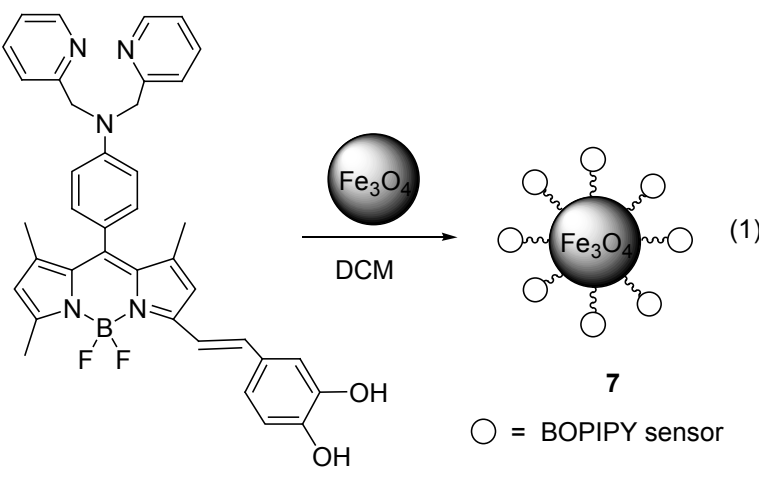

等 ${ }^{[9]}$ 报道了一种具有良好水溶性的 $\mathrm{Zn}^{2+}$ 比率苂光探针 8(图 10). 其光谱性质是在 HEPES 缓冲溶液中测试的, 加入 $\mathrm{Zn}^{2+}$ 后, 探针 $661 \mathrm{~nm}$ 处的荧光强度下降, $521 \mathrm{~nm}$ 处 的荧光强度上升, $I_{521} / I_{661}$ 从 0.3 升至 2.1, 实现对 $\mathrm{Zn}^{2+}$ 的 检测. 探针在水溶液中的溶解度达到 $50 \mathrm{mg} / \mathrm{mL}$, 对碱 金属和碱土金属的离子不敏感, 只有同为反磁性的 $\mathrm{Ni}^{2+}, \mathrm{Co}^{2+}$ 和 $\mathrm{Cu}^{2+}$ 能够淬灭探针苂光. 由于 $2,2^{2}$-二甲基 吡啶胺的存在, 探针只能在 $\mathrm{pH}>4$ 的环境中工作. 在 SW620 细胞的苂光成像实验中, 外源 $\mathrm{Zn}^{2+}$ 在羟基吡啶 硫酮的帮助下穿过细胞膜，使探针苂光由红变绿.

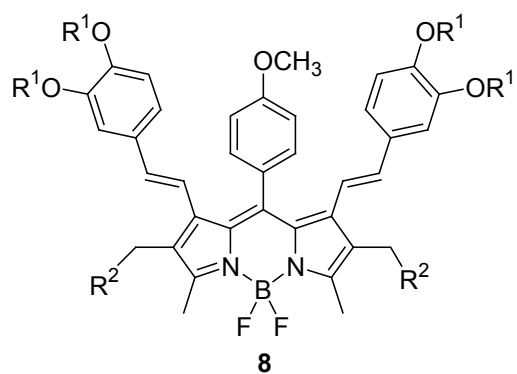<smiles>[R1]CCC(COCCOCCOCCOC)COCCOCCOCCOC</smiles>

图 10 水溶性 BODIPY 类 $\mathrm{Zn}^{2+}$ 探针 8

Figure 10 Water-soluble $\mathrm{Zn}^{2+}$ sensor based on BODIPY

\subsection{BODIPY 荧光探针检测 $\mathrm{Hg}^{2+}$}

$\mathrm{Hg}^{2+}$ 是一种极具生理毒性的化学物质, 是最严重的 重金属污染物之一, 开发响应快、易实现，能用于自然 环境和生物体系的新型 $\mathrm{Hg}^{2+}$ 检测方法是非常有意义的 工作. 2012 年, Vedamalai 等 ${ }^{[10]}$ 报道了一种以三唑为配体 的近红外 $\mathrm{Hg}^{2+}$ 探针 9(图 11), 由于 ICT 作用的存在, 空 探针的荧光被淬灭; 加入 $\mathrm{Hg}^{2+}$ 后探针的吸收波长蓝移 约 $29 \mathrm{~nm}$, 在 $650 \mathrm{~nm}$ 处苂光强度增至原先的 65 倍, 溶液

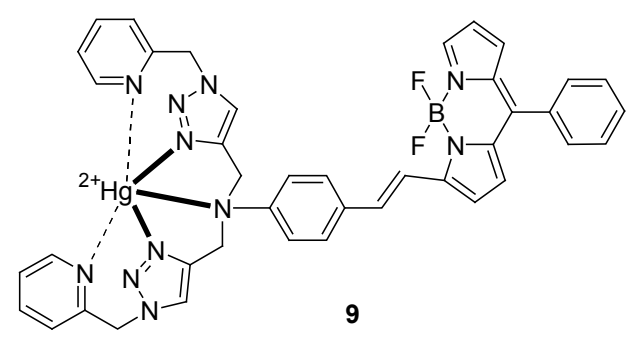

图 11 近红外 BODIPY $\mathrm{Hg}^{2+}$ 探针 9

Figure 11 NIR BODIPY $\mathrm{Hg}^{2+}$ sensor 9

则由蓝色变为紫色, 实现了对 $\mathrm{Hg}^{2+}$ 的比色-荧光双重检 测, 但探针在碱性条件下无法识别 $\mathrm{Hg}^{2+}$.

同年, Guo 等 ${ }^{[12]}$ 设计了具有对称 donor-acceptordonor 结构的近红外 $\mathrm{Hg}^{2+}$ 探针 $\mathbf{1 0}$ (Eq. 2), 其光谱性质是 在 $\mathrm{CH}_{3} \mathrm{CN}-\mathrm{HEPES}$ 溶液中测试的, 由于 PET 作用导致空 探针苂光被淬灭, 络合 $\mathrm{Hg}^{2+}$ 后 $655 \mathrm{~nm}$ 处苂光增强至原 先的 30 倍, 但没有明显的光谱位移. 相比之前的探针, 该探针的录离子检出限 $(3 \mu \mathrm{g} / \mathrm{L})$ 大大提高, 并成功实现 了 $\mathrm{HeLa}$ 细胞中的共聚焦荧光成像.

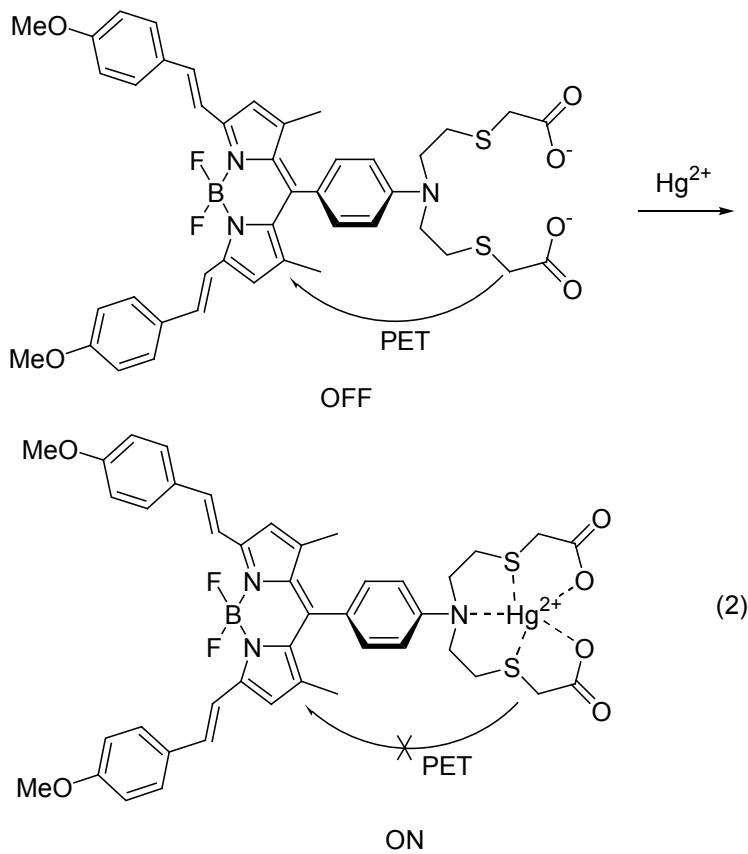

设计多功能 BODIPY 荧光探针已成为目前荧光探 针研究的热点之一. 2012 年, Qian 等 ${ }^{[12]}$ 报道了首例能够 同时响应 $\mathrm{Hg}^{2+}$ 和 $\mathrm{Ag}^{+}$的双通道探针 11 (Eq. 3). 加入 $\mathrm{Hg}^{2+}$ 后，1,3-丙二硫醇对醛基的保护被解除，探针吸收 波长蓝移 $16 \mathrm{~nm}$, 溶液颜色由浅粉色快速变为黄色, 用 肉眼即可分辨; 加入 $\mathrm{Ag}^{+}$后探针在 $550 \mathrm{~nm}$ 处的荧光增 强了 1 倍, 但没有明显的光谱位移, 其它金属离子对于 $\mathrm{Hg}^{2+}$ 和 $\mathrm{Ag}^{+}$几乎没有干扰.

2013 年, Zhang 等 ${ }^{[13]}$ 利用硫代冠醚对 $\mathrm{Hg}^{2+}$ 的选择性 螯合，设计合成了基于 ICT 机理的荧光探针 12(图 12). 
<smiles></smiles>

11

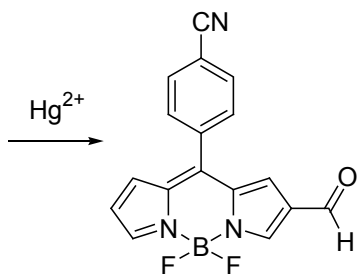

(3)

探针的光谱性质是在 $V\left(\mathrm{CH}_{3} \mathrm{CN}\right): V(\mathrm{HEPES})=4: 1$ 溶 液中测试的, 由于 $\mathrm{Hg}^{2+}$ 的鳌合降低了 $\mathrm{N}$ 原子的电子云 密度, ICT 效应被抑制, 探针 $594 \mathrm{~nm}$ 处的吸收峰消失, $564 \mathrm{~nm}$ 处出现新吸收峰, 与此同时, 探针的发射波长蓝 移约 $39 \mathrm{~nm}, I_{617} / I_{710}$ 从 0.8 升至 $3.5, I_{617} / I_{710}$ 在加入其它金 属阳离子时均无明显变化, 检测下限达到 $4.36 \mathrm{nmol} / \mathrm{L}$. 另外, 探针在 $\mathrm{HeLa}$ 细胞内成功实现了对 $\mathrm{Hg}^{2+}$ 的激光共 聚焦成像.

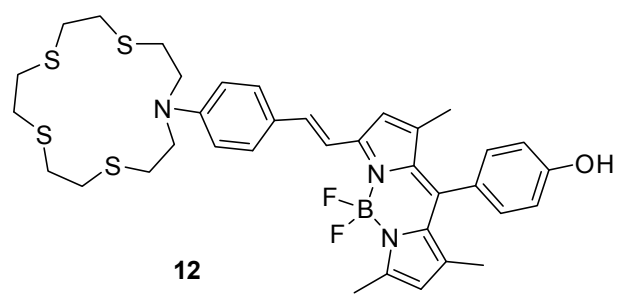

图 12 高灵敏度 BODIPY $\mathrm{Hg}^{2+}$ 探针 $\mathbf{1 2}$

Figure 12 Highly sensitive BODIPY $\mathrm{Hg}^{2+}$ sensor 12

\subsection{BODIPY 荧光探针检测 $\mathrm{Fe}^{3+}$}

铁离子是人体内含量最丰富的过渡金属离子, 在许 多生化反应中扮演重要角色. 2012 年 Wang 等 ${ }^{\left[{ }^{[14]}\right.}$ 设计合 成了一种基于 PET 机理的 $\mathrm{Fe}^{3+}$ 探针 BOD-NHOH 13 (Eq. 4). 无 $\mathrm{Fe}^{3+}$ 时, PET 作用将探针荧光淬灭; 加入 $\mathrm{Fe}^{3+}$ 后, $\mathrm{Fe}^{3+}$ 选择性氧化羟胺基团, $615 \mathrm{~nm}$ 处探针苂光增至原先 的 35 倍. 由于羟胺基团的存在, 该探针的水溶性良好, 并实现了 MCF-7 细胞中对外加 $\mathrm{Fe}^{3+}$ 的激光共聚焦成像. 由于羟胺基团在酸性环境中容易质子化, 所以该探针只 能在生理 $\mathrm{pH}(6.8 \sim 7.8)$ 条件下使用.
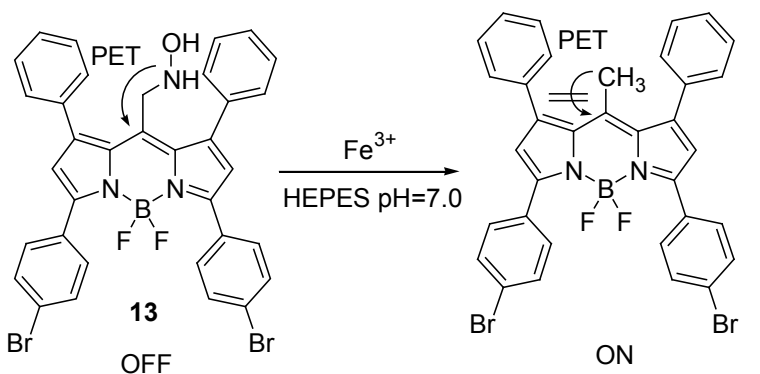

(4)

同年, $\mathrm{Qu}$ 等 ${ }^{[15]}$ 报道了应用 TBET (Through-Bond Energy Transfer)机理的 $\mathrm{Fe}^{3+}$ 探针 14(图 13). 使用 $334 \mathrm{~nm}$ 的光进行激发时, 通过能量转移, 探针荧光峰出现在 $732 \mathrm{~nm}$ 处, 红移达 $398 \mathrm{~nm}$, 能量传递效率超过 99\%. 加

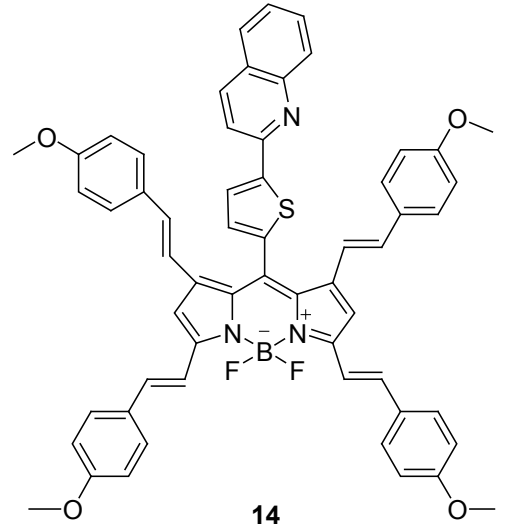

图 13 基于 TBET 机理的 BODIPY $\mathrm{Fe}^{3+}$ 探针 14

Figure 13 TBET-based BODIPY $\mathrm{Fe}^{3+}$ sensor 14

入 $\mathrm{Fe}^{3+}$ 后, 能量转移被抑制, 探针的荧光被淬灭, 加入 其他金属阳离子均对苂光强度没有影响. MCF-7 细胞的 荧光共聚焦实验证实了探针对 $\mathrm{Fe}^{3+}$ 的苂光标记功能.

\subsection{BODIPY 荧光探针检测 $\mathrm{Pb}^{2+}$}

铅离子是一种对生物体特别是人体危害很大的重 金属离子. 2009 年 Song 等 ${ }^{[16]}$ 设计合成了以另一种以纳 米粒子为载体的 $\mathrm{Pb}^{2+}$ 苂光探针 15 (Eq. 5), 以纳米 $\mathrm{Fe}_{3} \mathrm{O}_{4}$ 粒子为核心, 在其表面覆盖一层 $\mathrm{SiO}_{2}$ 后, 将 BODIPY 苂 光探针通过共价键连接到磁性纳米粒子表面. 在加入 $\mathrm{Pb}^{2+}$ 后, PET 过程受阻, 探针由浅蓝色转为浅粉色, 荧光 强度增至原先的 100 倍, 最低检测浓度达到 $1.5 \mu \mathrm{g} / \mathrm{L}$. 另 外，由于采用 $\mathrm{Fe}_{3} \mathrm{O}_{4}$ 作为纳米粒子的核心，探针复合体 具有良好的水溶性并且可以通过磁铁进行回收而实现 循环利用.
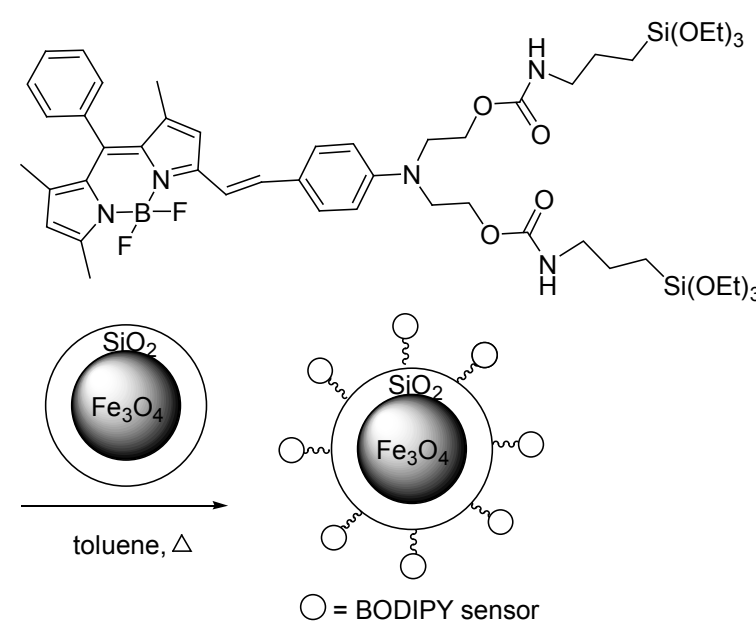

15

2012 年, Park 等 ${ }^{[17]}$ 报道了 $\mathrm{Pb}^{2+}$ 比率荧光探针 $16(\mathrm{Eq}$. 6). 在含 $1 \% \mathrm{CH}_{3} \mathrm{CN}$ 的 HEPES 溶液中加入 $\mathrm{Pb}^{2+}$ 后, 探针 $496 \mathrm{~nm}$ 处的吸收峰逐渐消失, 同时 $527 \mathrm{~nm}$ 处出现新吸 收峰, $I_{527} / I_{496}$ 不断增大, 溶液颜色由黄转粉, 值得注意 
的是, 探针和 $\mathrm{Pb}^{2+}$ 的络合是可逆的，随着 $\mathrm{Pb}^{2+}$ 的加入探 针 $-\mathrm{Pb}^{2+}$ 复合物以粉色固体形式析出, 再加入少量 $\mathrm{H}^{+}$或 EDTA 后粉色固体又重新溶于水中. 该探针的检测下限 达到了 $1 \mu \mathrm{mol} / \mathrm{L}$, 但灵敏度受到溶液 $\mathrm{pH}$ 的影响, 适合 在生理 $\mathrm{pH}$ 范围工作.

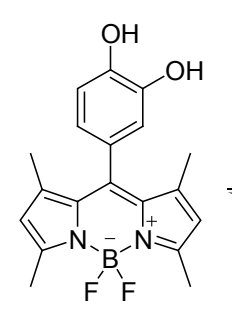

16
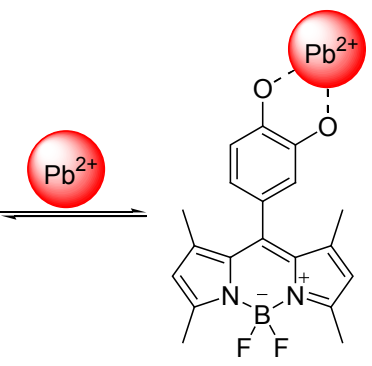

(6)

与水溶性纳米粒子担载 BODIPY 探针不同的是, 2013 年 Liu 等 ${ }^{[18]}$ 报道了水溶性的 OFF-ON 型 $\mathrm{Pb}^{2+}$ 探针 17(图 14). 探针的光谱性质是在中性 PBS 缓冲溶液中测 试的, 无 $\mathrm{Pb}^{2+}$ 存在时, 探针 $510 \mathrm{~nm}$ 处的苂光被 PET 作 用淬灭; 加入 2 倍当量的 $\mathrm{Pb}^{2+}$ 后, $510 \mathrm{~nm}$ 处苂光强度增 至原先 19 倍, 且不受其它金属阳离子的干扰. 探针荧光 强度与 $\mathrm{Pb}^{2+}$ 浓度在 $0.05 \sim 1.25 \mu \mathrm{mol} / \mathrm{L}$ 范围内成正比，检 测下限达到 $13.4 \mathrm{nmol} / \mathrm{L}$, 这也是迄今为止报道的灵敏 度较高的 $\mathrm{Pb}^{2+}$ 探针. 滴定实验证实了探针和 $\mathrm{Pb}^{2+}$ 是以 $1: 2$ 螯合的, 但螯合物的荧光强度受到 $\mathrm{pH}$ 的影响.<smiles>Cc1cc(C)n(P)c1-c1ccc(N(CC(=O)N(CCO)CCO)CC(=O)N(CCO)CCO)c(N(CC(=O)N(CCO)CCO)CC(=O)N(CCO)CCO)c1</smiles>

17

图 14 水溶性 BODIPY $\mathrm{Pb}^{2+}$ 探针 17

Figure 14 BODIPY $\mathrm{Pb}^{2+}$ sensor soluble in water

\subsection{BODIPY 荧光探针检测 $\mathrm{Cu}^{2+}$}

铜元素是自然界和生物环境中一类非常重要的元 素, 在人体、植物和动物等的体内各种生物代谢中发挥 着关键作用, 然而当其在体内和环境中富集到一定程度 时, 对很多生物体是污染性和致毒性的. 但是由于铜离 子很容易与水作用形成水合物, 而水合物的酸性性质使 得用有机溶剂的 $\mathrm{pH}$ 缓冲液很难检测出铜离子. Zhao 等 ${ }^{[19]}$ 报道了基于 6 -羟基吲哚的开关型荧光探针 BODIPY-EP (18) (Eq. 7). 当存在 $\mathrm{Cu}^{2+}$ 时, $\mathrm{Cu}^{2+}$ 能够选择
性地催化探针内酯键的水解, 释放出 2-吡啶甲酸. 在这 一过程中, 探针的苂光量子产率由 0.07 升至 $0.33,577$ $\mathrm{nm}$ 处苂光强度增至原先的 5 倍. 在生理 $\mathrm{pH}$ 附近, 探针 的水解在 $10 \mathrm{~min}$ 内即可完成, 对于其它在生物体内存在 的阳离子具有良好的选择性, 并在 HEK293A 细胞系中 实现了对外加 $\mathrm{Cu}^{2+}$ 的细胞成像.

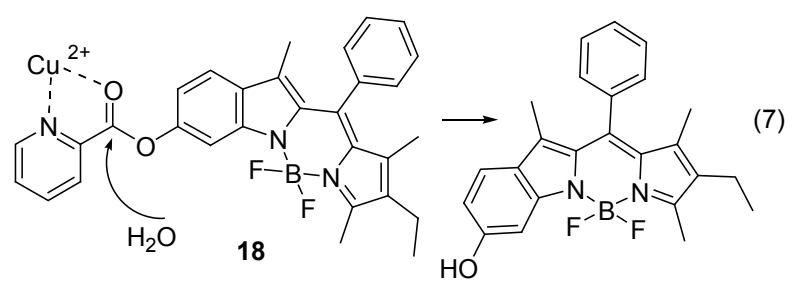

Yin 等 ${ }^{[20]}$ 设计合成了荧光波长位于近红外区的 $\mathrm{Cu}^{2+}$ 探针 19 (图 15). 在纯 $\mathrm{CH}_{3} \mathrm{CN}$ 中加入 $\mathrm{Cu}^{2+}$ 后, 探针 位于 $525 \mathrm{~nm}$ 的吸收峰消失同时在 $636 \mathrm{~nm}$ 处形成新的吸 收峰, 溶液颜色由粉变紫. 加入 6 倍当量 $\mathrm{Cu}^{2+}$ 后探针 $652 \mathrm{~nm}$ 处的苂光增强了约 250 倍, 体现了探针的高灵敏 度, 但遗憾的是, 当 $\mathrm{CH}_{3} \mathrm{CN}$ 溶液含水量超过 $10 \%$ 后, $\mathrm{Cu}^{2+}$-探针复合物的苂光就会被淬灭, 说明探针还无法 水溶液中工作, 此外 $\mathrm{Ag}^{+}$也对 $\mathrm{Cu}^{2+}$ 的检测形成干扰.

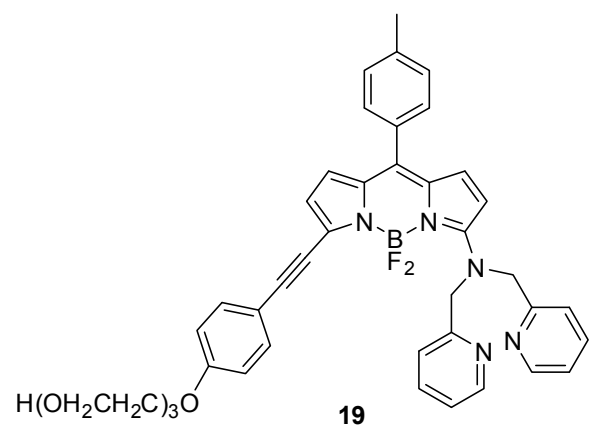

图 15 近红外 BODIPY $\mathrm{Cu}^{2+}$ 探针 $\mathbf{1 9}$

Figure 15 NIR BODIPY $\mathrm{Cu}^{2+}$ sensor 19

2013 年, $\mathrm{Li}$ 等 ${ }^{[21]}$ 报道了基于 $2,2^{\prime}$-二甲基吡啶胺的 ON-OFF 型 $\mathrm{Cu}^{2+}$ 探针 $\mathbf{2 0}$ (图 16). 在 $V$ (Tris)： $V(\mathrm{DMSO})=9: 1$ 溶液中加入 $\mathrm{Cu}^{2+}$ 后, 探针的吸收峰蓝 移，溶液由绿转粉，同时探针在 $510 \mathrm{~nm}$ 处的苂光被淬<smiles></smiles>

图 16 高灵敏度 $\mathrm{Cu}^{2+}$ 探针 $\mathbf{2 0}$

Figure 16 Highly sensitive BODIPY $\mathrm{Cu}^{2+}$ sensor 20 
灭. 在常见金属阳离子中只有 $\mathrm{Ni}^{2+}$ 和 $\mathrm{Co}^{2+}$ 对探针产生 了轻微干扰, 探针的检测下限达到 $2.78 \mu \mathrm{mol} / \mathrm{L}$. HepG-2 细胞的抑制增生与荧光成像实验分别证实了该探针具 有较低的细胞毒性和穿透细胞膜的能力. 值得注意的 是，探针能够在弱酸性环境中工作，对 $\mathrm{H}^{+}$并不敏感.

同年，笔者等 ${ }^{[22]}$ 以咪唑为配体，设计合成了能同时 检测 $\mathrm{Cu}^{2+}$ 和 $\mathrm{Hg}^{2+}$ 的 BODIPY 探针 21(图 17). 探针的光 谱性质是在 $V\left(\mathrm{H}_{2} \mathrm{O}\right): V\left(\mathrm{CH}_{3} \mathrm{CN}\right)=2: 8$ 溶液中测试的, 加入 $\mathrm{Cu}^{2+}$ 时, 溶液由黄色变为咖啡色, $30 \mathrm{~min}$ 后又变为 无色, 探针的吸收光谱几乎完全消失, 同时荧光强度降 至原先的三分之一; 加入 $\mathrm{Hg}^{2+}$ 时, 探针的吸收光谱略微 红移, 溶液由黄转粉, 其它金属离子对探针均无干扰作 用.

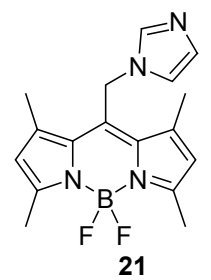

图 $17 \mathrm{Cu}^{2+}$ 和 $\mathrm{Hg}^{2+}$ 的双通道 BODIPY 探针 21

Figure 17 Dual-mode BODIPY sensor 21 for $\mathrm{Cu}^{2+}$ and $\mathrm{Hg}^{2+}$

另外, 笔者等 ${ }^{[23]}$ 也设计合成了 BODIPY-Cyclene 分 子 22(图 18), 此化合物在最大激发波长为 $517 \mathrm{~nm}$, 最大 发射波长为 $525 \mathrm{~nm}$, 其中只有 $\mathrm{Cu}^{2+}$ 对探针有荧光淬灭 效果. 同时测定了 $\mathrm{Cu}^{2+}$ 在不同浓度下对该探针的梯度 滴定变化, 并且发现该分子探针与 $\mathrm{Cu}^{2+}$ 的络合可能是 按 $1: 1$ 的最大比例进行络合. 从此变化中发现此探针 可能是基于 PET 的荧光识别机理, 此化合物具有极好的 水溶性, 可在水溶液中对 $\mathrm{Cu}^{2+}$ 进行检测.

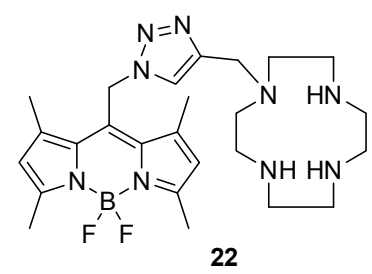

图 18 水溶性 BODIPY-cyclen $\mathrm{Cu}^{2+}$ 探针 $\mathbf{2 2}$

Figure 18 Water-soluble BODIPY-cyclen sensor 22 for $\mathrm{Cu}^{2+}$

2014 年, $\mathrm{Ye}$ 等 ${ }^{[24}$ 利用 $\mathrm{Cu}^{2+}$ 对腙的催化水解, 设计 合成了一款比率苂光探针 23 (Eq. 8). 探针的性质是在 $V\left(\mathrm{CH}_{3} \mathrm{CN}\right): V\left(\mathrm{H}_{2} \mathrm{O}\right)=1: 1$ 的溶液中测试的, 空白探针 没有苂光, 加入 $\mathrm{Cu}^{2+}$ 后, 探针 $525 \mathrm{~nm}$ 处的吸收峰逐渐 消失, 同时在 $490 \mathrm{~nm}$ 处出现新吸收峰, 溶液颜色由紫转 黄, 不受其它金属离子的干扰. 用 $460 \mathrm{~nm}$ 的光进行激发 时, 探针 $507 \mathrm{~nm}$ 处的荧光强度在加入 40 equiv. 的 $\mathrm{Cu}^{2+}$ 后增强了约 290 倍. 另外, 探针的灵敏度受到溶液酸碱
度的影响, 适合在 $\mathrm{pH}=6 \sim 8$ 范围工作. ${ }^{1} \mathrm{H}$ NMR 证实了 $\mathrm{Cu}^{2+}$ 催化下 $\mathrm{C}=\mathrm{N}$ 键的水解, HeLa 细胞的苂光成像实验 则说明了探针具有穿透细胞膜的能力.

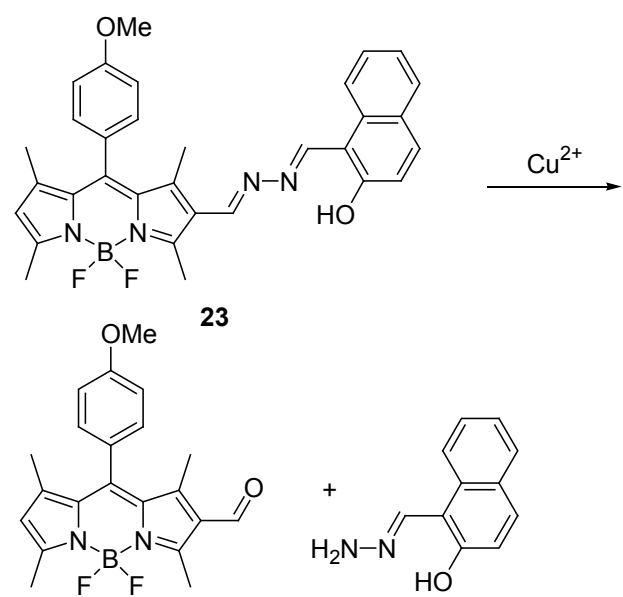

$\mathrm{He}$ 等 ${ }^{[25]}$ 报道了另一款能同时检测 $\mathrm{Cu}^{2+}$ 和 $\mathrm{Hg}^{2+}$ 的双 通道 BODIPY 探针 24(图 19). 空白探针在纯 $\mathrm{CH}_{3} \mathrm{CN}$ 中 有两处吸收峰, 分别位于 525 和 $350 \mathrm{~nm}$, 加入 $\mathrm{Hg}^{2+}$ 后, 原来的两个吸收峰分别红移至 571 和 $408 \mathrm{~nm}$, 溶液颜色 由粉转紫, $590 \mathrm{~nm}$ 处的苂光最大增强 5 倍, 检测下限达 到 $0.53 \mu \mathrm{mol} / \mathrm{L}$; 类似的, 加入 $\mathrm{Cu}^{2+}$ 后, 原来的两个吸收 峰红移至 $589 \mathrm{~nm}$ 和 $404 \mathrm{~nm}$, 溶液由粉转蓝, $610 \mathrm{~nm}$ 处 苂光最大增强 13 倍，检测下限达到 $0.08 \mu \mathrm{mol} / \mathrm{L}$. 其它 常见金属阳离子对探针均不形成干扰，值得注意的是， $\mathrm{Hg}^{2+}$ 和探针之间的螯合可逆，加入 EDTA 或半胱氨酸都 能够将探针复原，但由于 $\mathrm{Cu}^{2+}$ 和探针之间的螯合常数 远大于 $\mathrm{Hg}^{2+}$ ，所以 $\mathrm{Cu}^{2+}$ 和探针之间的螯合不可逆.

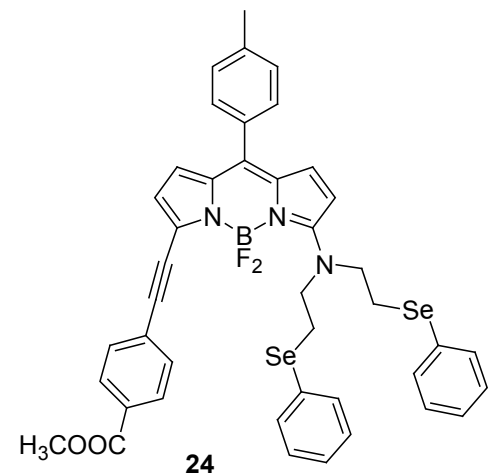

图 19 含硒双通道 BODIPY 探针 24

Figure 19 Dual-mode BODIPY Se-containing sensor $\mathbf{2 4}$ for $\mathrm{Cu}^{2+}$ and $\mathrm{Hg}^{2+}$

\subsection{BODIPY 荧光探针检测 $\mathrm{Cu}^{+}$}

2010 年, Domaille 等 ${ }^{[26]}$ 设计了一种能选择性识别 $\mathrm{Cu}^{+}$的比率苂光探针 25 (图 20), 该探针在 $480 \mathrm{~nm}$ 处被激 发, 络合 $\mathrm{Cu}^{+}$之后, 苂光强度之比 $\left(I_{560} / I_{490}\right)$ 从 2 变为大约 20 , 比例增大了 10 倍, 探针的苂光强度则增至原先的 


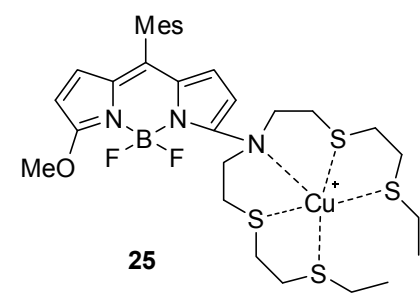

图 20 BODIPY $\mathrm{Cu}^{+}$探针 $\mathbf{2 5}$

Figure 20 BODIPY sensor 25 for $\mathrm{Cu}^{+}$

20 倍. 另外, 他们对 HEK293T 细胞进行了共聚焦苂光 成像实验, 证实了其具有响应活细胞内 $\mathrm{Cu}^{+}$浓度变化的 能力.

\subsection{BODIPY 荧光探针检测 $\mathrm{Cd}^{2+}$}

镉被广泛地用于工业生产(如电池工业), 在磷肥中 也大量存在, 环境中镉能引起多种癌症发病率的增加. 人们急需各种改进的方法来探测各种环境样品、工业废 水以及生物组织里的 $\mathrm{Cd}^{2+}$ 含量. 2008 年 Cheng 等 ${ }^{[27]}$ 合成 了用于检测 $\mathrm{Cd}^{2+}$ 的水溶性 BODIPY 探针 26(图 21). 当 存在 $\mathrm{Cd}^{2+}$ 时, $\mathrm{Cd}^{2+}$ 与聚酰胺基团络合, PET 过程受阻, 探 针苂光强度升至原先的 195 倍, 检出限低至 $6 \times 10^{-7}$ $\mathrm{mol} / \mathrm{L}$. 另外, 该探针对环境 $\mathrm{pH}$ 呈现很强的惰性, 苂光 发射强度与 $\mathrm{Cd}^{2+}$ 的浓度呈线性关系, 有望实现对 $\mathrm{Cd}^{2+}$ 的定量分析. 由于聚酰胺基团和氰基的引入，该探针水 溶性良好, 并很好地解决了生物背景苂光干扰的问题.

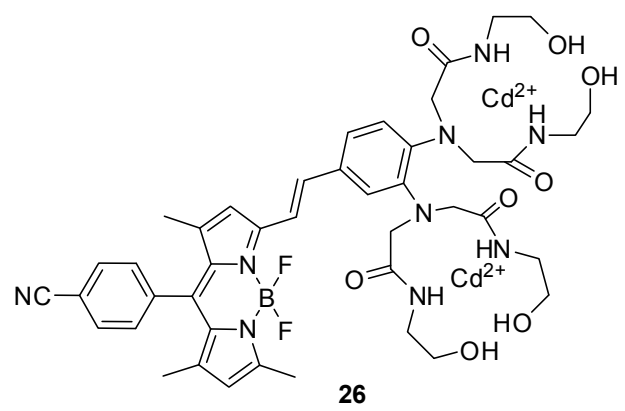

图 21 水溶性 BODIPY $\mathrm{Cd}^{2+}$ 探针 $\mathbf{2 6}$

Figure 21 Water-soluble BODIPY sensor 26 for $\mathrm{Cd}^{2+}$

2012 年, Pamuk 等 ${ }^{[28]}$ 报道了一种 $\mathrm{Cd}^{2+}$ 诱导的 BODIPY 氧化型 PET 苂光开关 27(图 22), 结合 $\mathrm{Cd}^{2+}$ 后

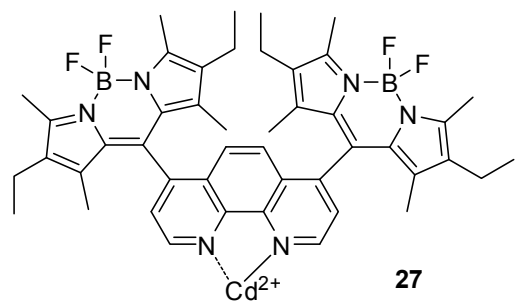

图 22 BODIPY 开关型 $\mathrm{Cd}^{2+}$ 探针 27

Figure 22 BODIPY fluorescent switch 27 for $\mathrm{Cd}^{2+}$
电子从 BODIPY 染料传递到邻菲罗啉上，苂光被淬灭; 加入三氟乙酸同样使得探针荧光淬灭, 形成了 NOR 型 $\mathrm{Cd}^{2+}$ 苂光逻辑门，其他离子对探针几乎没有影响.

\subsection{BODIPY 荧光探针检测 $\mathrm{K}^{+}$}

$\mathrm{K}^{+}$对于维持细胞渗透平衡、酸碱平衡以及传导神 经冲动起着重要作用. 2011 年, Hirata 等 ${ }^{[29]}$ 报道了首例 可用肉眼识别变化的 $\mathrm{K}^{+}$探针 28 (图 23), 其光谱性质是 在 HEPES-乙腈溶液中测试的, 加入钾离子后探针吸收 波长蓝移 $20 \mathrm{~nm}, 580 \mathrm{~nm}$ 处苂光峰强度增至原先的 100 倍, 溶液由蓝色变为紫色. 过量的 $\mathrm{Na}^{+}$对该探针几乎没 有干扰，长波激发也减少了对细胞的损伤.

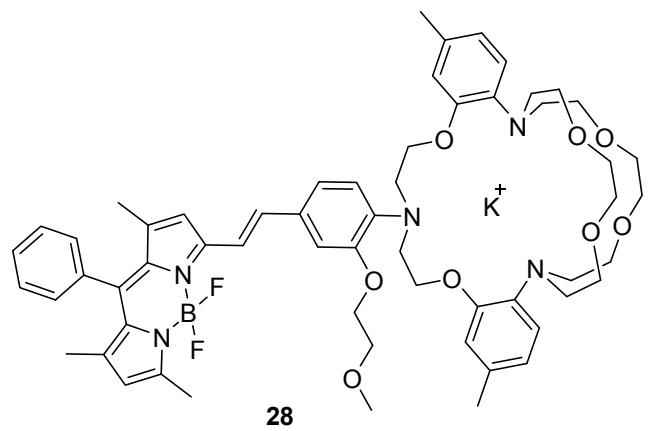

图 23 BODIPY $\mathrm{K}^{+}$荧光传感器 $\mathbf{2 8}$

Figure 23 BODIPY sensor 28 for $\mathrm{K}^{+}$

\subsection{BODIPY 荧光探针检测 $\mathrm{Au}^{3+} / \mathrm{Au}^{+}$}

2012 年, Wang 等 ${ }^{[30]}$ 报道了一种反应型 $\mathrm{Au}^{3+} / \mathrm{Au}^{+}$探 针 29 (Eq. 9), 由于有效抑制了 PET 过程, 加入 5 equiv. 的 $\mathrm{Au}^{3+}$ 后探针 $511 \mathrm{~nm}$ 处苂光强度增至原先的 116 倍. 相比之前报道的探针，该探针对 $\mathrm{Au}^{3+}$ 的检测下限更低 (63 $\mu \mathrm{g} / \mathrm{L})$, 响应时间更短 $(<60 \mathrm{~min})$, 且细胞渗透性良
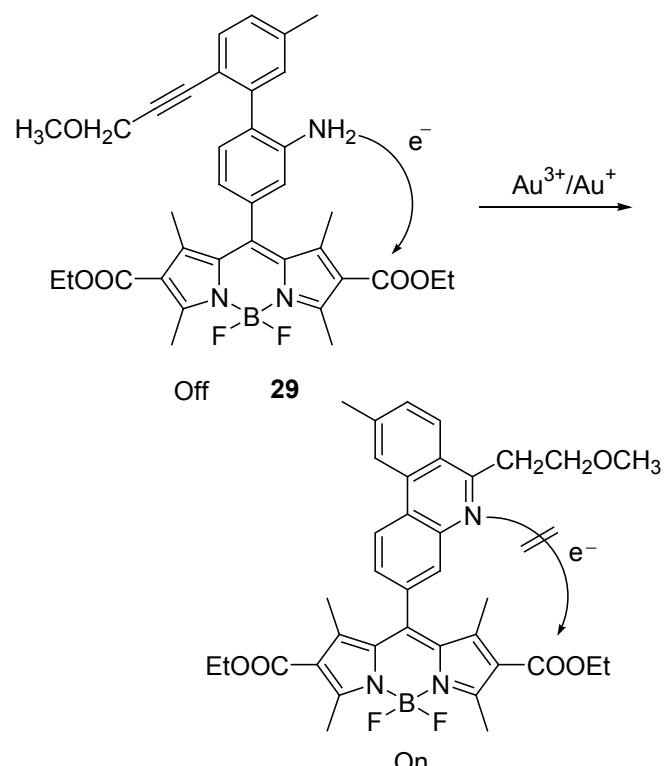
好, 在 HeLa 细胞中成功实现了对 $\mathrm{Au}^{3+}$ 的激光共聚焦成 像.

同年, Park 等 ${ }^{[31}$ 将 HEPES 还原 $\mathrm{Au}^{3+}$ 与金纳米粒子 对 $\mathrm{C}$ - I 键的选择性断裂相结合, 设计合成了一种反应 型 $\mathrm{Au}^{3+}$ 探针 30 (Eq. 10). 在没有金纳米粒子存在的情况 下, 探针荧光由于重原子效应的存在而被淬灭, 加入 $300 \mu \mathrm{mol} / \mathrm{L}$ 的 $\mathrm{Au}^{3+}$-HEPES 溶液后, 探针 $510 \mathrm{~nm}$ 处的苂 光在 $30 \mathrm{~min}$ 内增强了 500 倍. 值得注意的是, 即使溶液 中含有 $\mathrm{Au}^{+}, \mathrm{HEPES}$ 缓冲液也无法将其还原为金纳米粒 子，体现了探针极佳的选择性.

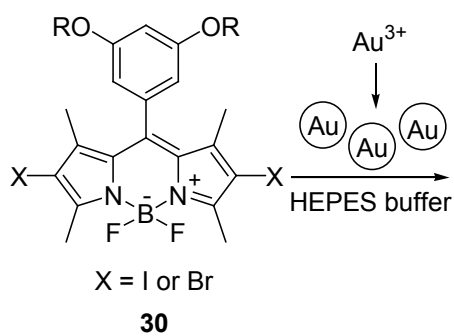

30

Weak Fluorescence<smiles>[R20]c1cc([R])cc(C(=C2C(C)=CC(C)=[N+]2P)c2c(C)c([TlH])c(C)n2P)c1</smiles>

$\mathrm{R}=\left(\mathrm{CH}_{2} \mathrm{CH}_{2} \mathrm{O}\right)_{3} \mathrm{CH}_{3}$ Strong Fluorescence
化合物 30 还可用于检测 $\mathrm{Pd}^{2+}{ }^{[32]}$ (Eq. 11), 在 $V(\mathrm{EtOH}): V\left(\mathrm{H}_{2} \mathrm{O}\right)=1: 4$ 的溶液中加入 $\mathrm{Pd}^{2+}$ 后, $\mathrm{Pd}^{2+}$ 被 $\mathrm{EtOH}$ 还原形成 $\mathrm{Pd}$ 纳米粒子, 催化了探针中 $\mathrm{C}-\mathrm{I}$ 键的 断裂, 使探针 $510 \mathrm{~nm}$ 处的菼光增强了超过 560 倍, 在 $10 \sim 50 \mu \mathrm{mol} / \mathrm{L}$ 的范围内苂光强度和 $\mathrm{Pd}^{2+}$ 成正比, 最低 检测浓度达到 $1 \mu \mathrm{g} / \mathrm{L}$.<smiles></smiles><smiles></smiles>

2.10 BODIPY 苂光探针检测 $\mathrm{Yb}^{3+}, \mathrm{Al}^{3+} / \mathrm{Cr}^{3+}$ 等

另外, 2012 年, $\mathrm{He}$ 等 ${ }^{[33]}$ 报道了近红外 $\mathrm{Yb}^{3+}$ 探针 31(图 24), 络合 $\mathrm{Yb}^{3+}$ 后, 探针复合体在 975 和 $1000 \mathrm{~nm}$ 处的荧光显著增强, 一方面可以检测稀土 $\mathrm{Yb}^{3+}$, 另一方 面提供了长波光激发下在近红外区发光的配合物, 可用 于生物医学成像和荧光免疫分析. 2014 年, BarbaBon 等 ${ }^{[34]}$ 报道了一款基于 ICT 机理, 能选择性检测 $\mathrm{Al}^{3+}$ 和 $\mathrm{Cr}^{3+}$ 的探针 32(图 24). 在 $V\left(\mathrm{H}_{2} \mathrm{O}\right): V\left(\mathrm{CH}_{3} \mathrm{CN}\right)=4: 6$ 的混合溶液中空白探针在 $603 \mathrm{~nm}$ 处有强吸收峰但苂光 被 ICT 作用淬灭, 加入 $\mathrm{Al}^{3+}$ 或 $\mathrm{Cr}^{3+}$ 后探针在 $560 \mathrm{~nm}$ 处 出现新吸收峰, 溶液由粉色转为蓝色, 同时 $563 \mathrm{~nm}$ 处探 针苂光增强约 30 倍, 包括 $\mathrm{Fe}^{3+}$ 在内的其他金属阳离子 对探针不形成干扰, 但遗憾的是, 探针只能识别 $\mathrm{Al}^{3+}$ 和 $\mathrm{Cr}^{3+}$ 的存在却没有办法区分两者.

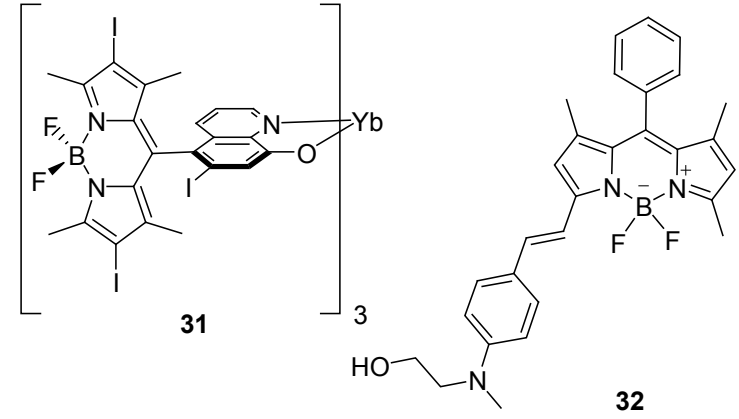

图 24 BODIPY $\mathrm{Yb}^{3+}$ 探针 $\mathbf{3 1}$ 和 $\mathrm{Al}^{3+} / \mathrm{Cr}^{3+}$ 探针 32

Figure 24 BODIPY probe for $\mathrm{Yb}^{3+} 31$ and for $\mathrm{Al}^{3+} / \mathrm{Cr}^{3+} 32$

\section{BODIPY 类荧光探针对阴离子的检测}

\subsection{BODIPY 荧光探针检测 $\mathrm{ClO}$}

次氯酸根离子在生物体防止外界微生物入侵方面 起着重要作用. 2011 年, $\mathrm{Kim}$ 等 ${ }^{[35]}$ 报道了一种 $\mathrm{ClO}^{-}$探针 33 (Eq. 12). 在中性缓冲溶液中, $\mathrm{ClO}^{-}$将硫醚氧化为亚 枫, $650 \mathrm{~nm}$ 处苂光峰消失的同时 $525 \mathrm{~nm}$ 处的苂光显著 增强. 在 RAW264.7 巨噬细胞中, 该探针实现了对于外 源性及内源性 $\mathrm{ClO}^{-}$浓度变化的检测，并在低浓度下对 巨噬细胞无毒，具有重要的生物学意义.

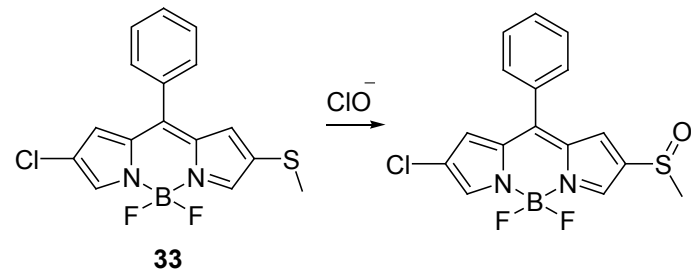

\subsection{BODIPY 荧光探针检测 $\mathrm{CN}$}

目前在设计 $\mathrm{CN}^{-}$探针时遇到的最大障碍来自于 $\mathrm{F}^{-}$ 的干扰. 2012 年, Lee 等 ${ }^{[36]}$ 报道了一种能够在水相中识 别 $\mathrm{CN}^{-}$的探针 34 (Eq. 13), 其光谱性质是在含水超过 $99 \%$ 的溶液中测试的，加入 $\mathrm{CN}^{-}$后 $520 \mathrm{~nm}$ 处苂光增强 约 43 倍, 相比之前报道的探针, 该探针不仅较好地解决 了 $\mathrm{F}^{-}$的干扰问题，还能够应用于生物检测，具有一定的 生物学意义.

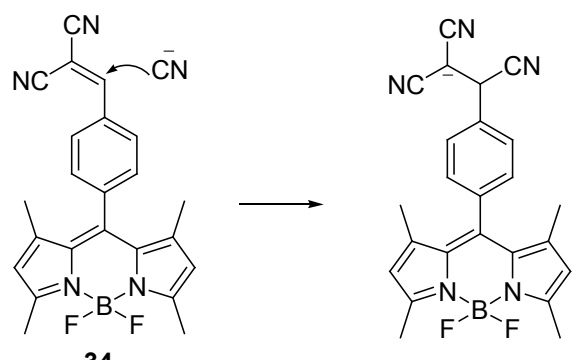

2013 年, Madhu 等 ${ }^{[37]}$ 设计合成了一种新型 $\mathrm{CN}^{-}$比率 
荧光探针 35 (Eq. 14), 在含水 $1 \%$ 的乙腈溶液中, $\mathrm{CN}^{-}$进 攻羰基形成氰醇, $554 \mathrm{~nm}$ 处的荧光被完全淬灭, 溶液则 由橘黄变为浅黄. $\mathrm{CN}^{-}$的浓度可由吸收光谱中 $486 / 538$ $\mathrm{nm}$ 处荧光强度之比得到, 其它阴离子对 $\mathrm{CN}^{-}$完全不存 在干扰.

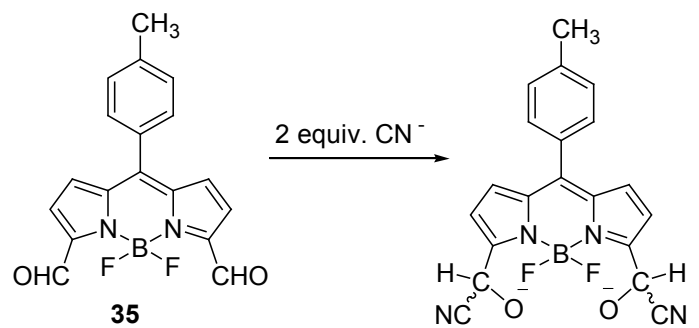

同年, Zhang 等 ${ }^{[38]}$ 报道了具备水溶性的 $\mathrm{CN}^{-}$比率荧 光探针 36 (Eq. 15). 结合 $\mathrm{CN}^{-}$后, $600 \mathrm{~nm}$ 处的苂光强度 下降, $550 \mathrm{~nm}$ 处的荧光强度迅速上升, 荧光强度之比 $\left(I_{550} / I_{600}\right)$ 增大了约 80 倍, 溶液由紫色变为粉色. 探针对 常见无机阴离子具有良好的选择性, 检测限达到 0.1 $\mu \mathrm{mol} / \mathrm{L}$

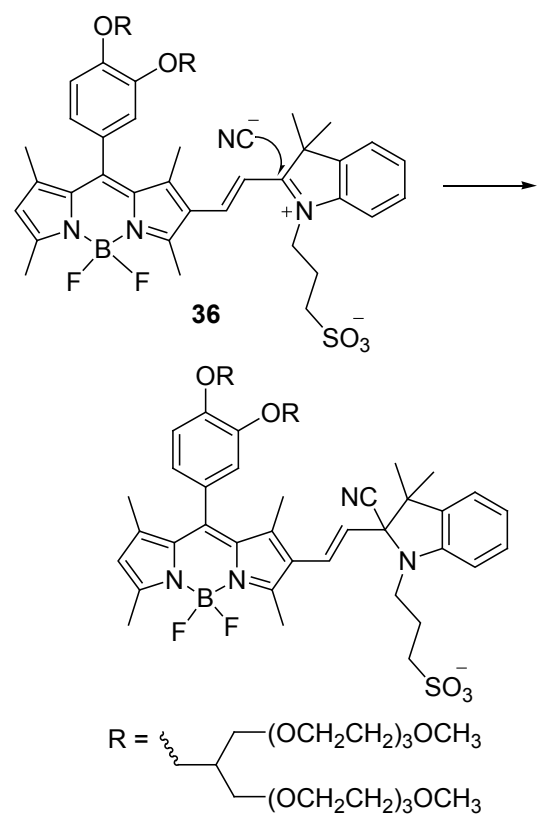

\subsection{BODIPY 荧光探针检测 $F^{-}$}

$\mathrm{F}^{-}$是最小的阴离子, 同时具有很高的水合热, 在生 物和环境中有重要作用, 其荧光检测具有挑战性. 2010 年, Bozdemir 等 ${ }^{[39]}$ 设计合成了两种分别基于 PET 和 ICT 机理的 $\mathrm{F}^{-}$探针 37 和 38(图 25). 在乙腈溶液中, $\mathrm{F}^{-}$能选择 性地切断探针 37 和 $\mathbf{3 8}$ 中的 $\mathrm{Si}-\mathrm{O}$ 键, $506 \mathrm{~nm}$ 处探针 37 的苂光强度减至原先的 $1 / 20$, 而探针 38 的最大吸收波 长则由 $560 \mathrm{~nm}$ 红移至 $682 \mathrm{~nm}$, 同时荧光被淬灭. 而且 探针 38 的发射和吸收波长均位于近红外区, 有效降低 了生物体背景荧光干扰，但存在响应时间过长的问题.

2011 年 $\mathrm{Fu}$ 等 ${ }^{[40]}$ 利用 $\mathrm{F}$ 和 $\mathrm{Si}$ 之间的强亲和性, 开发
出一种新型 $\mathrm{F}^{-}$荧光探针 39 (Eq. 16). 加入 TBAF 后, $\mathrm{F}^{-}$ 选择性切断探针中的 $\mathrm{Si}-\mathrm{C}$ 键, 释放出三己基氟化硅, 同时探针的苂光发射波长由 $571 \mathrm{~nm}$ 蓝移至 $554 \mathrm{~nm}$, 颜 色由橙黄色转为绿色. 与之前报道的 $\mathrm{F}^{-}$探针相比, 该探 针的最低检测浓度达 $1.28 \mu \mathrm{g} / \mathrm{L}$, 响应时间小于 $5 \mathrm{~min}$, 但其光谱性质是在丙酮中测试的, 仍不具备良好的水溶 性.
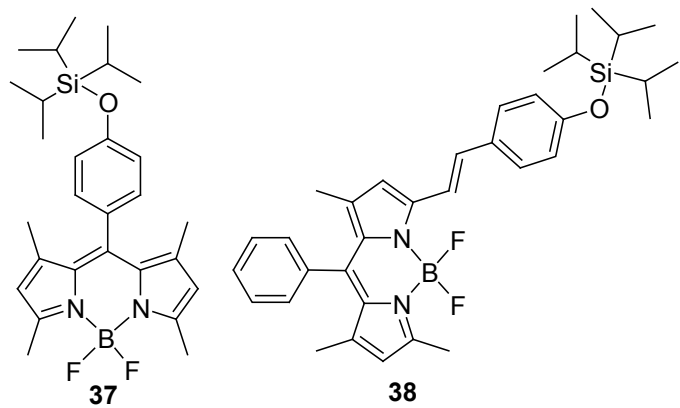

图 25 BODIPY $\mathrm{F}^{-}$探针 $\mathbf{3 7}$ 和探针 $\mathbf{3 8}$

Figure 25 BODIPY sensor 37 and sensor 38 for $\mathrm{F}^{-}$

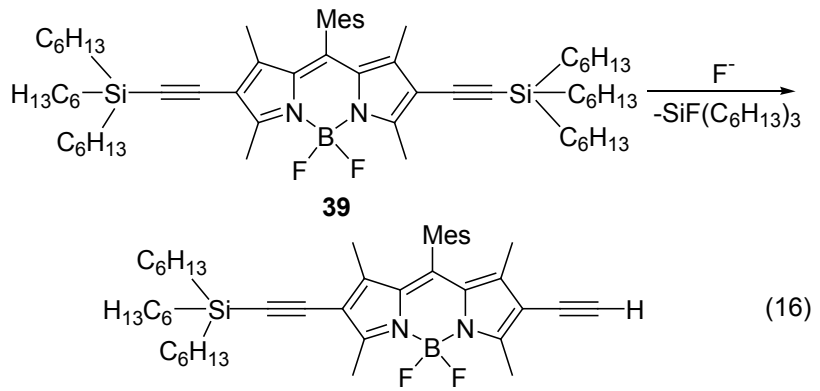

2012 年, Guliyev 等 ${ }^{[41]}$ 报道了一种可同时检测 $\mathrm{F}^{-}$和 $\mathrm{CN}^{-}$的类 BODIPY 探针 40(图 26), 探针母核中六元环被 七元环取代, 其光谱性质是在氯仿中测试的, 加入 $\mathrm{F}^{-}$或 $\mathrm{CN}^{-}$后都能使探针的吸收波长大幅红移, 但 $\mathrm{F}^{-}$红移更 为明显, 溶液由黄变紫, 而 $\mathrm{CN}^{-}$则使溶液由黄变红. 多 功能荧光探针是当前研究热点, 将该探针负载至聚合物 基底上有望制成多功能阴离子检测芯片.

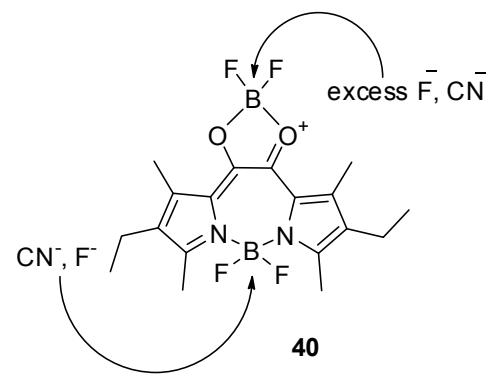

图 26 双通道类 BODIPY 探针 $\mathbf{4 0}$

Figure 26 Dual-mode pseudo-BODIPY sensor for $\mathrm{F}^{-}$and $\mathrm{CN}^{-}$

2013 年, Swamy 等 ${ }^{[42]}$ 报道了一种基于 FRET 机理的 双荧光 $\mathrm{F}^{-}$探针 41(图 27), 空探针在 502 和 $335 \mathrm{~nm}$ 处存 
在双重荧光, 加入 TBAF 后, B 变为 4 配位, 空间构型的 变化导致从硼烷到 BODIPY 的能量传递效率大幅降低, $515 \mathrm{~nm}$ 处的荧光被完全淬灭, 实现对 $\mathrm{F}^{-}$的检测.

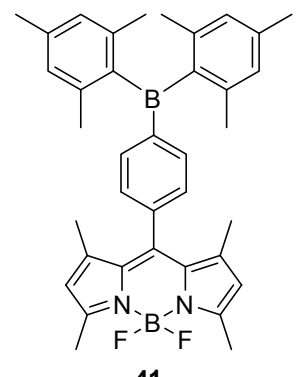

41

图 27 基于 FRET 机理的 BODIPY 探针 $\mathbf{4 1}$

Figure 27 FRET-based BODIPY sensor $\mathbf{4 1}$ for $\mathrm{F}^{-}$

Sarkar 等 ${ }^{[43]}$ 报道了另一种同样基于 FRET 机理, 但 却具有三重苂光波长的 $\mathrm{F}^{-}$探针 42(图 28). 探针的共轭 连接臂是由噻吩构成的, 由于噻吩的稳定化能比苯环 低，因此探针分子内能量传递的效率更高. 在 $\mathrm{CH}_{2} \mathrm{Cl}_{2}$ 中, 在 $350 \mathrm{~nm}$ 处激发时, 探针出现三处苂光峰, 分别位 于 404,535 和 $613 \mathrm{~nm}$; 当用 $510 \mathrm{~nm}$ 的光激发时, 出现 两个苂光峰, 535 和 $613 \mathrm{~nm}$. 加入 TBAF 后, 探针原先的 两个吸收峰蓝移, 在 $624 \mathrm{~nm}$ 处出现新吸收峰, 溶液由红 色变为深绿. 与此同时, 探针 $606 \mathrm{~nm}$ 处的菼光强度下 降, $520 \mathrm{~nm}$ 处的苂光逐渐增强, 形成对 $\mathrm{F}^{-}$的比率苂光响 应，常见阴离子对检测均不存在干扰.

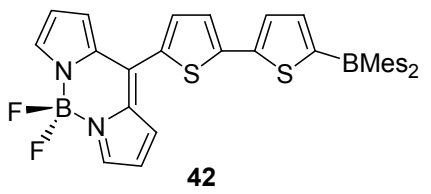

图 28 三重苂光 BODIPY 探针 42

Figure 28 Tricolour emission BODIPY sensor $\mathbf{4 2}$ for $\mathrm{F}^{-}$

2014 年, Mahapatra 等 ${ }^{[44]}$ 报道了一款 $\mathrm{F}^{-}$比率荧光探 针 43 (Scheme 2). 该探针的光谱性质是在 $V\left(\mathrm{CH}_{3} \mathrm{CN}\right)$ : $V\left(\mathrm{H}_{2} \mathrm{O}\right)=7: 3$ 的溶液中测试的, 加入 TBAF 后, 探针 $350 \mathrm{~nm}$ 处的吸收峰逐渐消失, 同时 419 和 $254 \mathrm{~nm}$ 处出 现新吸收峰, 溶液颜色由浅黄色转为深咖啡色, 在 UV 照射下, 溶液原本绿色的苂光变为深蓝色. 在加入 $\mathrm{F}^{-}$的 过程中, $\mathrm{N}$ 原子上的氢先是和 $\mathrm{F}^{-}$形成氢键, 而后过量的 $\mathrm{F}^{-}$则将 $\mathrm{N}$ 原子去质子化, ${ }^{1} \mathrm{H} \mathrm{NMR}$ 证实了上述检测机理. 值得注意的是, $\mathrm{AcO}^{-}$同样能够脱去氮原子上的质子, 但 由于碱性不够强的缘故, 溶液颜色只是由浅黄色变为红 色, 不对 $\mathrm{F}^{-}$产生干扰. RAW 细胞中的苂光成像证明了 了探针穿透细胞膜的能力.
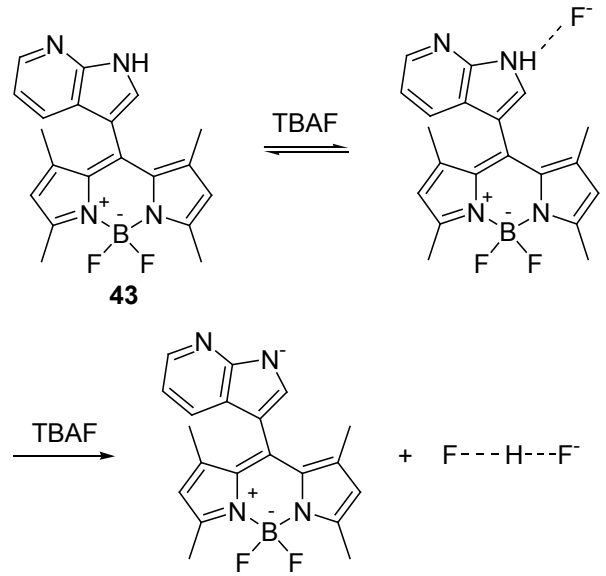

Scheme 2

\subsection{BODIPY 荧光探针检测 $\mathrm{SO}_{3}{ }^{2-}$}

2012 年, $\mathrm{Gu}$ 等 ${ }^{[45]}$ 报道了一种用于检测 $\mathrm{SO}_{3}^{2-}$ 的双重 比率荧光探针 44 (Eq. 17), 在 $V\left(\mathrm{H}_{2} \mathrm{O}\right): V(\mathrm{DMSO})=1: 1$ 溶液中加入 $\mathrm{SO}_{3}^{2-}$ 后, 探针吸收波长由 $510 \mathrm{~nm}$ 逐步红移 至 $620,570 \mathrm{~nm}$ 处, 苂光峰消失的同时在 $647 \mathrm{~nm}$ 处生成 新的荧光峰, 利用加入 $\mathrm{SO}_{3}^{2-}$ 前后吸收强度之比/荧光 发射强度之比可以有效测定 $\mathrm{SO}_{3}^{2-}$ 的浓度, 其它常见阴 离子对探针几乎没有干扰, $\mathrm{SO}_{3}^{2-}$ 检出限达 $5.8 \times 10^{-5}$ $\mathrm{mol} / \mathrm{L}$.

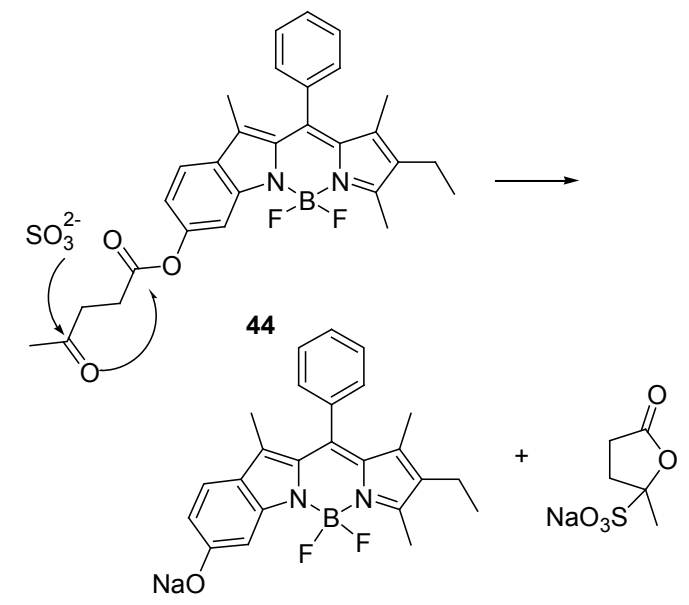

\subsection{BODIPY 荧光探针检测 $\mathrm{HSO}_{4}{ }^{-}$}

2012 年, $\mathrm{Li}$ 等 ${ }^{[46]}$ 报道了一种基于希夫碱的 OFF-ON 型 $\mathrm{HSO}_{4}^{-}$探针 45 (Scheme 3). 在 $\mathrm{CH}_{3} \mathrm{CN}$ 中，与苯环直接 相连的 $\mathrm{N}$ 原子通过 PET 作用淬灭探针荧光, 加入 $\mathrm{HSO}_{4}^{-}$后, 探针吸收光谱中 $324 \mathrm{~nm}$ 处的吸收峰消失, 在 $394 \mathrm{~nm}$ 处形成新的吸收峰, 溶液颜色由黄转绿, 同时 $\mathrm{HSO}_{4}^{-}$中的羟基和 $\mathrm{N}$ 原子之间形成氢键，抑制了 $\mathrm{PET}$ 作 用, $515 \mathrm{~nm}$ 处的苂光增强了约 20 倍. 将溶剂换成 $V\left(\mathrm{CH}_{3} \mathrm{CN}\right): V\left(\mathrm{H}_{2} \mathrm{O}\right)=1: 1$ 后, 其它阴离子对探针几乎 没有影响, 吸收光谱无明显变化, 但 $\mathrm{HSO}_{4}^{-}$电离出的 $\mathrm{H}^{+}$将 $\mathrm{N}$ 原子质子化, $512 \mathrm{~nm}$ 处的苂光增强了 6 倍. 探针 
在 $\mathrm{pH} 5 \sim 12$ 范围内均可工作且不受包括 $\mathrm{H}_{2} \mathrm{PO}_{4}{ }^{-}$在内的 阴离子干扰.

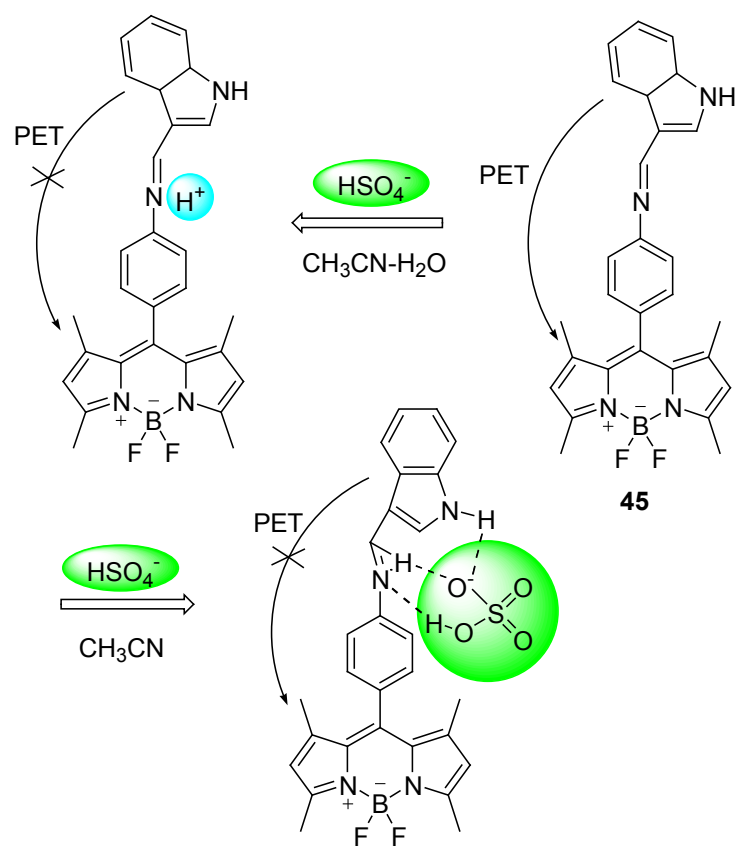

Scheme 3

同年, $\mathrm{Li}$ 等 ${ }^{[47]}$ 又设计合成了另一种基于席夫碱，能 够检测 $\mathrm{F}^{-}$和 $\mathrm{HSO}_{4}{ }^{-}$的双通道 BODIPY 探针 46 (Eq. 18). 探针的光谱性质仍然是在 $\mathrm{CH}_{3} \mathrm{CN}$ 中测试的, 没有加入 离子前, 探针在 399 和 $498 \mathrm{~nm}$ 处各有一个吸收峰, 加入 $\mathrm{F}^{-}$后, $399 \mathrm{~nm}$ 处的吸收峰逐渐消失, 同时在 $560 \mathrm{~nm}$ 处出 现新吸收峰, 溶液颜色由黄变紫, 检测下限达到 0.35 $\mu \mathrm{mol} / \mathrm{L}$. 探针 $518 \mathrm{~nm}$ 处的荧光被淬灭, 常见阴离子对 $\mathrm{F}^{-}$没有干扰. 与加入强碱 TBAOH 之后现象的对比, 证 明了荧光的淬灭来源于 $\mathrm{F}^{-}$对亚氨基的去质子化. 加入 $\mathrm{HSO}_{4}{ }^{-}$后亚氨基又重新被质子化, 探针荧光恢复, 形成 了 ON-OFF-ON 开关响应, 根据这种性质, 该探针有望 作为 “分子键盘锁” 进行信息加密.
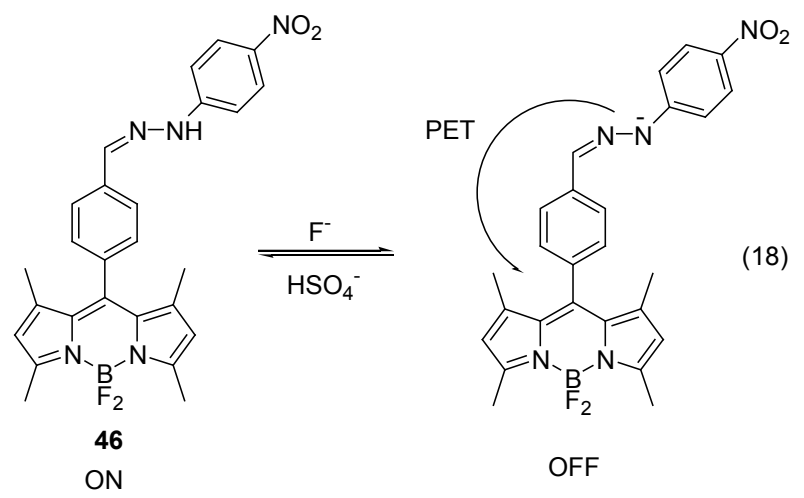

\section{4 结论与展望}

综上所述, 通过对 BODIPY 共轭骨架的修饰, 引入
不同的检测基团可得到具有不同功能的 BODIPY 类荧 光探针, 实现对各类离子的特异性识别. 随着研究的深 入，这类苂光探针已经显示出广阔的应用前景. 尽管部 分产品已通过荧光成像技术用于活细胞中离子和分子 的识别检测，但 BODIPY 在离子探针的应用方面，对阴 离子检测的灵敏度和选择性还远不如阳离子; 在生物检 测方面, 大多数人工合成的 BODIPY 染料都不溶于水, 且发射波长小于 $600 \mathrm{~nm}$, 背景干扰较大, 在一定程度上 限制了这种苂光染料的生物应用. 为了得到性能更为理 想的苂光探针, 需要发展高收率便捷的有机合成新方法 来对其结构进行化学修饰，得到吸收和发射波长均在近 红外或红外区的荧光探针. 因此, 合成具有高灵敏度、 高选择性、生物相容性好、发射波长位于近红外或红外 区，且能够在生物体内实现苂光成像的 BODIPY 类荧光 探针将会是未来研究热点和发展方向.

\section{References}

[1] Treibs, A.; Kreuzer, F. H. Justus Liebigs Ann. Chem. 1968, 718, 208.

[2] (a) Boens, N.; Leen, V.; Dehaen, W. Chem. Soc. Rev. 2012, 41, 1130.

(b) Ulrich, G.; Ziessel, R.; Harriman, A. Angew. Chem., Int. Ed. 2008, 47,1184 .

(c) Loudet, A; Burgess, K. Chem. Rev. 2007, 107, 4891.

(d) Ziessel, R.; Ulrich, G. Harriman A New J. Chem. 2007, 31, 496.

(e) Kamkaew, A.; Lim, S. H.; Lee, H. B.; Kiew, L. V.; Chung, L. Y.; Burgess, K. Chem. Soc. Rev. 2013, 42, 77.

[3] Han, J. Y.; Loudet, A.; Barhoumi, R.; Burghardt, R.; Burgess, K. J. Am. Chem. Soc. 2009, 131, 1642.

[4] Boens, N.; Qin, W. W.; Baruah, M.; De Borggraeve, W. M.; Filarowski, A.; Smisdom, N.; Ameloot, M.; Crovetto, L.; Tlavera, E. M.; Alvarez-Pez, J. M. Chem. Eur. J. 2011, 17, 10924.

[5] Chen, Y. T.; Wang, H. L.; Wan, L.; Bian, Y. Z.; Jiang, J. Z. J. Org. Chem. 2011, 76, 3774.

[6] Hecht, M.; Krausb, W.; Rurack, K. Analyst 2013, 138, 325.

[7] Cao, J.; Zhao, C. C.; Wang, X. Z.; Zhang, Y. F.; Zhu, W. H. Chem. Commun. 2012, 48, 9897.

[8] Kang, G.; Son, H. J.; Lim, J. M.; Kweon, H. S.; Lee, I. S.; Kang, D. M.; Jung, J. H. Chem. Eur. J. 2012, 18, 5843.

[9] Zhu, S. L.; Zhang, J. T.; Janjanam, J.; Vegesna, G.; Luo, F. T.; Tiwari, A.; Liu, H. Y. J. Mater. Chem. B 2013, 1, 1722.

[10] Vedamalai, M.; Wu, S. P. Org. Biomol. Chem. 2012, 10, 5410.

[11] Zhao, Y.; Lv, X.; Liu, Y, L.; Liu, J.; Zhang, Y.; Shi, H. P.; Guo, W. J. Mater. Chem. 2012, 22, 11475.

[12] Zhang, X. J.; Xu, Y. F.; Guo, P.; Qian, X. H. New J. Chem. 2012, $36,1621$.

[13] Zhang, T. P.; She, G. W.; Qi, X. P.; Mu, L. X. Tetrahedron 2013, 69, 7102 .

[14] Wang, R.; Yu, F. B.; Liu, P.; Chen, L. X. Chem. Commun. 2012, $48,5310$.

[15] Qu, X. Y.; Liu, Q.; Ji, X. N.; Chen, H. C.; Zhou, Z. K.; Shen, Z. Chem. Commun. 2012, 48, 4600.

[16] Lee, H. Y.; Bae, D. R.; Park, J. C.; Song, H.; Han, W. S.; Jung, J. H. Angew. Chem., Int. Ed. 2009, 48, 1239.

[17] Park, J.; Kim, Y. Analyst 2012, 137, 3246.

[18] Liu, J.; Wu, K.; Li, S.; Song, T.; Han, Y. F.; Li, X. Dalton Trans. 
2013, 42, 3854.

[19] Zhao, C. C.; Feng, P.; Cao, J.; Wang, X. Z.; Yang, Y.; Zhang, Y. L.; Zhang, J. X.; Zhang, Y. F. Org. Biomol. Chem. 2012, 10, 3104.

[20] Yin, S. C.; Yuan, W. L.; Huang, J. L.; Xie, D. B.; Liu, B.; Jiang, K. Z. Qiu, H. Y. Spectrochim. Acta, Part A 2012, 96, 82.

[21] Li, Z.; Chen, Q. Y.; Wang, P. D.; Wu, Y. RSC Adv. 2013, 3, 5524.

[22] Xie, R. J.; Yi, Y. R.; He, Y.; Liu, X. G.; Liu, Z. X. Tetrahedron 2013, 69, 8541.

[23] Yi, Y. R. M.S. Thesis, Zhejiang Univesity, Hangzhou, 2011 (in Chinese). (易云瑞，硕士论文，浙江大学，杭州, 2011).

[24] Ye, J. H.; Xu, J.; Chen, H. C.; Bai, Y.; Zhang, W. C.; He, W. J. RSC Adv. 2014, 4, 6691.

[25] He, X. X.; Zhang, J.; Liu, X. G.; Dong, L.; Li, D.; Qiu, H. Y.; Yin, S. C. Sens. Actuators, B 2014, 192, 29.

[26] Domaille, D.; Zeng, L.; Chang, C. J. J. Am. Chem. Soc. 2010, 132, 1194.

[27] Cheng, T. Y.; Xu, Y. F.; Zhang, S. Y.; Zhu, W. P.; Qian, X. H.; Duan, L. P. J. Am. Chem. Soc. 2008, 130, 16160.

[28] Pamuk, M.; Algi, F. Tetrahedron Lett. 2012, 53, 7010.

[29] Hirata, T.; Terai, T.; Komatsu, T.; Hanaoka, K.; Nagano, T. Bioorg. Med. Chem. Lett. 2011, 21, 6090.

[30] Wang, J. B.; Wu, Q. Q.; Min, Y. Z.; Liu, Y. Z.; Song, Q. H. Chem. Commun. 2012, 48, 744.

[31] Park, J.; Choi, S.; Kim, T.; Kim, Y. Analyst 2012, 137, 4411.

[32] Keum, D.; Kim, S. W.; Kim, Y. M. Chem. Commun. 2014, 50, 1268.

[33] He, H. S.; Si, L. P.; Zhong, Y. H.; Dubey, M. Chem. Commun.
2012, 48, 1886.

[34] Barba-Bon, A.; Calabuig, L.; Costero, A. M.; Gil, S.; Martinez-Manez, R.; Sancenon, F. RCS Adv. 2014, 4, 8962.

[35] Kim, T-I.; Park, S.; Choi, Y. D.; Kim, Y. M. Chem. Asian J. 2011, $6,1358$.

[36] Lee, C. H.; Yoon, H. J.; Shim, J. S.; Jang, W. D. Chem. Eur. J. 2012, 18, 4513.

[37] Madhu, S.; Basu, S. K.; Jadhav, S.; Ravikanth, M. Analyst 2013, 138, 299.

[38] Zhang, J. T.; Zhu, S. L.; Valenzano, L.; Luo, F. T.; Liu, H. Y. RSC Adv. 2013, 3, 68 .

[39] Bozdemir, O. A.; Sozmen, F.; Buyukcakir, O.; Guliyev, R.; Cakmak, Y.; Akkaya, E. U. Org. Lett. 2010, 12, 1400.

[40] Fu, L.; Jiang, F. L.; Fortin, D.; Harvey, P. D.; Liu, Y. Chem. Commun. 2011, 47, 5503.

[41] Guliyev, R.; Ozturk, S.; Sahin, E.; Akkaya, E. U. Org. Lett. 2012, $14,1528$.

[42] Swamy P, C. A.; Mukherjee, S.; Thilagar, P. Chem. Commun. 2013, 49, 993.

[43] Sarker, S. K.; Thilagar, P. Chem. Commun. 2013, 49, 8558.

[44] Mahapatra, A. J.; Maji, R.; Maiti, K.; Adhikari, S. S.; Mukhopadhyay, C. D.; Mandal, D. Analyst 2014, 139, 309.

[45] Gu, X. F.; Liu, C. H.; Zhu, Y. C.; Zhu, Y. Z. J. Agric. Food Chem. 2011, 59, 11935 .

[46] Li, Q.; Guo, Y.; Shao, S. J. Analyst 2012, 137, 4497.

[47] Li, Q.; Yue, Y.; Guo, Y.; Shao, S. J. Sens. Actuators, B 2012, 173, 797. 